\title{
Genome-wide mapping of QTL associated with heterosis in the RIL-based NCIII design
}

\author{
HE XiaoHong ${ }^{1}$, HU ZhongLi ${ }^{2} \&$ ZHANG Yuan-Ming ${ }^{1 *}$ \\ ${ }^{1}$ Section on Statistical Genomics, State Key Laboratory of Crop Genetics and Germplasm Enhancement/Chinese National Center for Soybean \\ Improvement, College of Agriculture, Nanjing Agricultural University, Nanjing 210095, China; \\ ${ }^{2}$ State Key Laboratory of Hybrid Rice, College of Life Sciences, Wuhan University, Wuhan 430072, China
}

Received October 29, 2011; accepted March 12, 2012; published online April 10, 2012

\begin{abstract}
Heterosis represents one of the most revolutionary advancements in crop improvement. In the genetic dissection of heterosis, NCIII design is one of the most powerful and widely used mating schemes. However, the methodologies for quantitative trait loci (QTL) detection in the design were mostly based on composite interval mapping. Therefore, in this study, our purpose was to develop a statistical method for mapping epistatic QTL associated with heterosis in the RIL-based NCIII design. First, we derivated the expectations of two classical linear transformations, $Z_{1}$ and $Z_{2}$, while a quantitative trait was controlled by two QTL with digenic epistasis and arbitrary linkage under the $\mathrm{F}_{\infty}$ and $\mathrm{F}_{2}$ metric models. Then, we constructed an epistatic genetic model that includes all markers on the whole genome simultaneously, and estimated all the parameters in the model by the empirical Bayes approach. Finally, a series of Monte Carlo simulation experiments was carried out to confirm the proposed approach. The results show that: (1) all the augmented genetic parameters for main-effect QTL could be rightly identified with satisfactory statistical power and precision; (2) the statistical powers in the detection of augmented epistatic effects were substantively affected by the signs of pure epistatic effects; (3) it is more difficult to detect epistatic QTL than to detect main-effect QTL; (4) statistical power is higher in the RIL-based NCIII design than in the $\mathrm{F}_{2}$-based NCIII design, especially in the detection of the augmented epistatic effect that consists of two pure epistatic effects in opposite directions.
\end{abstract}

empirical Bayes, epistasis, NCIII design, heterosis, quantitative trait locus, recombinant inbred line

Citation: He X H, Hu Z L, Zhang Y M. Genome-wide mapping of QTL associated with heterosis in the RIL-based NCIII design. Chin Sci Bull, 2012, 57: 2655-2665, doi: 10.1007/s11434-012-5127-x

Heterosis, or hybrid vigor, results in the phenotypic superiority of a hybrid over its parents with respect to traits such as growth rate, reproductive success, and yield [1]. This phenomenon has been recognized since ancient civilization [2-4], under scientific investigation since Darwin [5] in the absence of genetics, and for over 100 years with genetic considerations [6,7]. Heterosis represents one of the most revolutionary advancements in crop improvement, i.e., maize [8] and rice [4]. In the genetic dissection of heterosis, NCIII design, proposed by Comstock and Robinson [9,10], is one of the most powerful and widely used mating schemes [11-17]. Therefore, the importance of heterosis

*Corresponding author (email: soyzhang@njau.edu.cn or soyzhang@ hotmail.com) necessitates an in-depth study of the NCIII design.

The NCIII design is to cross the $i$ th individual $(i=1,2, \ldots$, $n$ ) of an $\mathrm{F}_{2}$ population to both parental lines $\left(\mathrm{P}_{1}\right.$ and $\left.\mathrm{P}_{2}\right)$ to produce $2 n$ families $\left(L_{i 1}\right.$ and $\left.L_{i 2}\right)$. An analysis of variance (ANOVA) of phenotypic means and differences between the backcross progenies provides estimates of additive and dominance genetic variances with nearly equal precision when epistasis is absent, and these variances are further used to estimate the average degree of dominance. However, the conventional ANOVA approach only deals with the collective effects of all the polygenes [18]. The introduction of molecular markers has facilitated the mapping of quantitative trait loci (QTL) in numerous species, and substantial progress has been achieved in the detection of individual 
QTL and their interaction for heterosis in yield and other traits in the NCIII design.

One modification of the NCIII design is to use $\mathrm{F}_{3}$ as the base population. The design was adopted by Stuber et al. [19] to study the genetic dissection of heterosis by mapping QTL for heterosis in grain yield and five other traits, along with single marker analysis [20] and interval mapping (IM) [21]. The results showed that overdominance (or pseudoover-dominance) plays a significant role in heterosis for the grain yield. Another modification of the NCIII design is to use recombinant inbred lines (RIL) as the base population. The results showed that the primary genetic basis of heterosis is dominance in the interval mapping by Xiao et al. [22], and epistasis and overdominance in the mixed model analysis [23] by Li et al. [24], Luo et al. [25] and Mei et al. [26]. It should be noted that repulsion linkage might lead to pseudo-overdominance. To overcome this issue, Lu et al. [12] developed the $\mathrm{F}_{2} \mathrm{Syn} 3$-based NCIII design and found that 24 QTL (86\%) for grain yield exhibited overdominance, while most of the QTL for plant height, grain moisture, and stalk lodging showed partial to complete dominance. QTL analyses were performed separately for the two backcross populations using composite interval mapping (CIM) [27] and the additive effect, dominance effect, and the level of dominance for a QTL were indirectly estimated according to the deduction of Cockerham and Zeng [11]. Using the method of Lu et al. [12], LeDeaux et al. [28] assessed the stability of QTL involved in the expression of heterosis in the RIL-based NCIII design, and the results showed that the yield QTL showed a positive dominant gene action, whereas the ear height and DPS QTL exhibited behavior that ranged from negative to positive dominance. Among these above designs, the RIL-based NCIII design is the best. The reasons are as follows. First, RIL is a permanent segregating population, so it can be shared between research groups, and testcross progeny can be repeatedly generated and phenotyped as needed $[14,15,26,29]$. Second, RIL allows more recombinational segregation of tightly linked QTL than the other populations, and this offers a higher mapping resolution [15,22,29]. Finally, the use of homozygous RIL can gain the highest statistical power for QTL detection $[15,17,29]$. Therefore, further exploration of the RIL-based NCIII design is warranted.

To date, several theoretical studies on the genetic dissection of heterosis have been carried out. Cockerham and Zeng [11] developed the theory and statistical analysis of orthogonal contrasts for a single marker locus to characterize the effects of QTL in the $\mathrm{F}_{2^{-}}$and $\mathrm{F}_{3}$-based NCIII designs. Four orthogonal contrasts, $C_{1}$ to $C_{4}$, among the means of progenies from marker genotype $\times$ parental line combinations are constructed, of which $C_{1}$ and $C_{3}$ provide estimates and tests for additive and dominance effects of linked QTL, respectively, whereas $C_{2}$ and $C_{4}$ for epistatic effects. Epistatic effects contribute to the additive effects in $C_{1}(a d$ and $d a)$ and the dominance effects in $C_{3}(a a)$, and distinct epistatic effects contribute to $C_{2}$ ( $a a$ and $d d$ ) and $C_{4}(a d$ and $d a$ ). The method is used to re-analyze the maize data of Stuber et al. [19]. The results showed that dominance can explain the observed heterosis in grain yield and other traits, although epistasis can also play an important role, and overdominance at individual QTL level cannot be ruled out. In addition, QTL effects in Stuber et al. [19] are $a-d$ and $a+d$ in the two backcross populations, respectively.

Melchinger et al. [14] developed a general theoretical framework of composite interval mapping for determining the contributions of different types of genetic effects to heterosis. They defined the augmented additive and dominance effects in the RIL-based NCIII design: the former measures the net contribution of the QTL to the parental difference and the latter measures the net contribution of the QTL to midparent heterosis (MPH).

Li et al. [15] studied heterosis by QTL analysis in the RIL-based NCIII design. QTL analysis was performed separately for the RIL, MPH of two backcross populations and the SUM $\left(L_{1}+L_{2}\right)$ and DIFF $\left(L_{1}-L_{2}\right)$ data of two backcross populations $\left(L_{1}\right.$ and $\left.L_{2}\right)$. In the absence of epistasis, the analysis of RIL and SUM datasets identifies QTL with additive effect, whereas the analysis of MPH and DIFF datasets detects QTL with dominance effect [30]. Main-effect QTL was detected in each mapping population by composite interval mapping [27] and digenic interaction was identified in each mapping population by the mixed linear approach [23]. The results suggested that heterosis was attributable to the orchestrated outcome of partial-to-complete dominance, overdominance, and epistasis.

However, these above methods were mostly based on composite interval mapping. Therefore, Garcia et al. [16] developed a multiple-interval mapping [31] model in the NCIII design. The method provides a platform to estimate the number, genomic positions, augmented additive and dominance effects, and epistatic interactions $(a a+d d$ and $a d+d a)$ of QTL simultaneously. The method was used to re-analyze the datasets in Stuber et al. [19] and Xiao et al. [22]. For the former, yield heterosis was mainly due to dominant gene action, although overdominance (or pseudo-overdominance) of individual QTL could not completely be ruled out. For the latter, the observed yield heterosis was mainly due to additive $\times$ additive epistatic effects but not dominant effects.

The objective of this study was to develop a statistical method for mapping epistatic QTL associated with heterosis in the RIL-based NCIII design. First, we calculated the genetic expectations of two classical linear transformations $Z_{1}$ and $Z_{2}$. Then, we constructed an epistatic genetic model that included all markers on the whole genome simultaneously and estimated all the parameters in the model by empirical Bayes of Xu [32]. Finally, a series of Monte Carlo simulation experiments was carried out to confirm the proposed approach. 


\section{Theory and methods}

\subsection{Genetic design and data collection}

An RIL population was derived by a single seed descent procedure from two inbred lines $\left(\mathrm{P}_{1}\right.$ and $\left.\mathrm{P}_{2}\right)$ that differed significantly in the quantitative traits of interest, and possessed abundant polymorphism molecular markers. A random sample of $n$ RIL individuals (female parents) was backcrossed to their two parental lines to produce $2 n$ families $\left(L_{1 i}\right.$ and $\left.L_{2 i}\right)$. All of the $2 n$ families, each with $m$ replications, were planted. Molecular marker information was observed from all of the $n$ RIL individuals, whereas quantitative traits were measured for all of the $2 \mathrm{~nm}$ NCIII progeny. The phenotypic observations were denoted by $y_{t i j}$, where $t=1$ and 2 for $L_{1}$ and $L_{2}$, respectively; $i=1,2, \ldots, n ; j=1,2, \ldots, m$. The family means were denoted by $\bar{L}_{t i}=\sum_{j=1}^{m} y_{t i j} / m$. Following Frascaroli et al. [30] and Melchinger et al. [14], we performed two linear transformations: $Z_{1 i}=\bar{L}_{1 i}+\bar{L}_{2 i}$ and $Z_{2 i}=\bar{L}_{1 i}-\bar{L}_{2 i}$. The association between $Z_{t}$ and the marker genotypes of the RIL lines were used to infer the genetic architecture of heterosis for the trait.

\subsection{Statistical genetic models for mapping heterotic QTL in RIL-based NCIII design}

The expected genetic values of $Z_{1 i}$ and $Z_{2 i}$ depended on the choice of the metric. Two main metrics were adopted for the populations derived from the cross between the two inbred lines: the $\mathrm{F}_{\infty}$ and $\mathrm{F}_{2}$ metrics [33-35]. The derivation of the expected genetic values of $Z_{1 i}$ and $Z_{2 i}$ under both the $\mathrm{F}_{\infty}$ and the $\mathrm{F}_{2}$ metric models is presented in Supplement Information A under the assumption that the quantitative trait was determined by two QTL with digenic epistasis and arbitrary linkage $(A$ and $B)$. The genetic effect symbols adopted in this research were described in Kao and Zeng [33].

(i) Statistical genetic models for mapping QTL in the RIL-based NCIII design under the $\mathrm{F}_{\infty}$ metric model. According to the expected genetic values of $Z_{1 i}$ under the $F_{\infty}$ metric model (Table S2), the phenotypic value of $Z_{1 i}$ can be described as

$$
\begin{aligned}
Z_{1 i}= & 2 \mu+x_{a_{1} i} a_{1}+d_{1}+x_{a_{2} i} a_{2}+d_{2}+x_{a_{1} a_{2} i} i_{a_{1} a_{2}} \\
& +x_{a_{1} d_{2} i} i_{a_{1} d_{2}}+x_{d_{1} a_{2} i} i_{d_{1} a_{2}}+x_{d_{1} d_{2} i} i_{d_{1} d_{2}}+e_{1 i},
\end{aligned}
$$

where $\mu$ is the mean genotypic values of the four homozygotes in RIL; $a_{k}$ and $d_{k}$ are additive and dominance effects of the $k$ th QTL $(k=1,2) ; i_{a_{1} a_{2}}, \quad i_{a_{1} d_{2}}, \quad i_{d_{1} a_{2}}$ and $i_{d_{1} d_{2}}$ are additivexadditive, additivexdominance, dominancexadditive and dominancexdominance epistatic effects (see Supplement Information A for details); $x_{a_{1} i}, x_{a_{2} i}, x_{a_{1} a_{2} i}, x_{a_{1} d_{2} i}$, $x_{d_{1} a_{2} i}$ and $x_{d_{1} d_{2} i}$ are determined by the genotype of the $i$ th RIL line (Table S2); and $e_{1 i}$ is the residual error with an $N\left(0, \sigma_{1}^{2}\right)$ distribution. According to the results in Table S2, the coefficients of the six genetic parameters have following restrictions: $x_{a_{1} a_{2} i}=x_{d_{1} d_{2} i}$ and $x_{a_{1} d_{2} i}=-x_{d_{1} a_{2} i}=\frac{1}{2}\left(x_{a_{1} i}-x_{a_{2} i}\right)$. To solve the genetic parameters, model (1) must be reduced to

$$
Z_{1 i}=\mu_{Z_{1}}+x_{a_{1} i} a_{1}^{*}+x_{a_{2} i} a_{2}^{*}+x_{\ddot{i}_{12} i} \vec{i}_{12}+e_{1 i}
$$

where $\mu_{Z_{1}}=2 \mu+d_{1}+d_{2}, a_{1}^{*}=a_{1}+\frac{1}{2}\left(i_{a_{1} d_{2}}-i_{d_{1} a_{2}}\right), a_{2}^{*}=a_{2}$ $+\frac{1}{2}\left(i_{d_{1} a_{2}}-i_{a_{1} d_{2}}\right), \vec{i}_{12}=\left(i_{a_{1} a_{2}}+i_{d_{1} d_{2}}\right)$ and $x_{\ddot{i}_{12} i}=x_{a_{1} a_{2} i}=x_{d_{1} d_{2} i}$.

If the quantitative trait was controlled by $q$ QTL, model (2) should be extended to

$$
Z_{1 i}=\mu_{Z_{1}}+\sum_{k=1}^{q} x_{a_{k}^{*} i} a_{k}^{*}+\sum_{k=1}^{q-1} \sum_{l=k+1}^{q} x_{\vec{i}_{l} l} \vec{i}_{k l}+e_{1 i}
$$

where the model mean $\mu_{z_{1}}=2 \mu+\sum_{k=1}^{q} d_{k} ; \quad a_{k}^{*}=a_{k}$ $+\frac{1}{2} \sum_{l \neq k}^{q}\left(i_{a_{k} d_{l}}-i_{d_{k} a_{l}}\right)$ is the augmented additive effect of QTL $k$, which measures the net contribution of the QTL to the parental difference (PD) [14,36]; and $\vec{i}_{k l}=i_{a_{k} a_{l}}+i_{d_{k} d_{l}}$ is augmented epistatic effect between QTL $k$ and $l$, which estimates the compounded effect of $a a$ and $d d$ epistatic effects between QTL $k$ and $l$. Coefficients $x_{a_{k}^{*} i}$ and $x_{\bar{i}_{k} i}$ are determined by genotypes of the $k$ th and $l$ th QTL (marker) for

\begin{tabular}{|c|c|c|c|c|c|c|c|c|c|c|c|c|}
\hline \multirow{3}{*}{ Marker genotype } & \multicolumn{6}{|c|}{$\mathrm{F}_{\infty}$ metric model } & \multicolumn{6}{|c|}{$\mathrm{F}_{2}$ metric model } \\
\hline & \multicolumn{3}{|c|}{$Z_{1 i}$} & \multicolumn{3}{|c|}{$Z_{2 i}$} & \multicolumn{3}{|c|}{$Z_{1 i}$} & \multicolumn{3}{|c|}{$Z_{2 i}$} \\
\hline & $x_{a_{k}^{*}}$ & $x_{a_{i}^{*}}$ & $x_{\ddot{i}_{k l}}$ & $u_{d_{k}^{*}}$ & $u_{d_{l}^{*}}$ & $u_{\tilde{i}_{k l}}$ & $x_{a_{k}^{*}}$ & $x_{a_{l}^{*}}$ & $x_{i_{k l}}$ & $u_{d_{k}^{*}}$ & $u_{d_{l}^{*}}$ & $u_{\tilde{i}_{k l}}$ \\
\hline$M_{k} M_{k} M_{l} M_{l}$ & 1 & 1 & 1 & -1 & -1 & 0 & 1 & 1 & 1 & -1 & -1 & $-1 / 2$ \\
\hline$M_{k} M_{k} m_{l} m_{l}$ & 1 & -1 & 0 & -1 & 1 & 1 & 1 & -1 & 0 & -1 & 1 & $1 / 2$ \\
\hline$m_{k} m_{k} M_{l} M_{l}$ & -1 & 1 & 0 & 1 & -1 & 1 & -1 & 1 & 0 & 1 & -1 & $1 / 2$ \\
\hline$m_{k} m_{k} m_{l} m_{l}$ & -1 & -1 & 1 & 1 & 1 & 0 & -1 & -1 & 1 & 1 & 1 & $-1 / 2$ \\
\hline
\end{tabular}
the $i$ th RIL line (Table 1).

Table 1 Coefficients of genetic parameters determined by marker genotypes of RIL line for the RIL-based NCIII $Z_{1 i}$ and $Z_{2 i}$ data under the $\mathrm{F}_{\infty}$ and $\mathrm{F}_{2}$ metric models 
In the same way, the phenotypic value of $Z_{2 i}$ can be described as

$$
\begin{aligned}
Z_{2 i}= & a_{1}+u_{d_{1} i} d_{1}+a_{2}+u_{d_{2} i} d_{2}+u_{a_{1} a_{2} i} i_{a_{1} a_{2}}+u_{a_{1} d_{2} i} i_{a_{1} d_{2}} \\
& +u_{d_{1} a_{2} i} i_{d_{1} a_{2}}+u_{d_{1} d_{2} i_{d_{1} d_{2}}}+e_{2 i},
\end{aligned}
$$

where $u_{d_{1} i}, u_{d_{2} i}, u_{a_{1} a_{2} i}, u_{a_{1} d_{2} i}, u_{d_{1} a_{2} i}$ and $u_{d_{1} d_{2} i}$ are determined by the genotype of the $i$ th RIL line (Table S2), and $e_{2 i}$ is the residual error with an $N\left(0, \sigma_{2}^{2}\right)$ distribution. According to the results in Table $\mathrm{S} 2$, there are $u_{a_{1} d_{2} i}=u_{d_{1} a_{2} i}$ and $u_{a_{1} a_{2} i}=-u_{d_{1} d_{2} i}=-\frac{1}{2}\left(u_{d_{1} i}+u_{d_{2} i}\right)$. To solve the genetic parameters, model (4) must be reduced to

$$
Z_{2 i}=\mu_{Z_{2}}+u_{d_{1} i} d_{1}^{*}+u_{d_{2} i} d_{2}^{*}+u_{\tilde{i}_{12}} \tilde{i}_{12}+e_{2 i}
$$

where $\quad \mu_{Z_{2}}=a_{1}+a_{2}, \quad d_{1}^{*}=d_{1}-\frac{1}{2}\left(i_{a_{1} a_{2}}-i_{d_{1} d_{2}}\right), \quad d_{2}^{*}=d_{2}$ $-\frac{1}{2}\left(i_{a_{1} a_{2}}-i_{d_{1} d_{2}}\right), \quad \tilde{i}_{12}=\left(i_{a_{1} d_{2}}+i_{d_{1} a_{2}}\right)$ and $u_{\tilde{i}_{12} i}=u_{a_{1} d_{2} i}=u_{d_{1} a_{2} i}$.

If the quantitative trait was controlled by $q$ QTL, model (5) should be extended to

$$
Z_{2 i}=\mu_{Z_{2}}+\sum_{k=1}^{q} u_{d_{k}^{*} i} d_{k}^{*}+\sum_{k=1}^{q-1} \sum_{l=k+1}^{q} u_{\tilde{i}_{k l} i} \tilde{i}_{k l}+e_{2 i}
$$

where the model mean $\mu_{z_{2}}=\sum_{k=1}^{q} a_{k} ; d_{k}^{*}=d_{k}-\frac{1}{2} \sum_{l \neq k}^{q}\left(i_{a_{k} a_{l}}\right.$ $\left.-i_{d_{k} d_{l}}\right)$ is the augmented dominance effect of QTL $k$, which measures the net contribution of the QTL to the midparent heterosis (MPH) [14,36]; and $\tilde{i}_{k l}=i_{a_{k} d_{l}}+i_{d_{k} a_{l}}$ is the augmented epistatic effect between QTL $k$ and $l$, which estimates the compounded effect of $a d$ and $d a$ epistatic effects between QTL $k$ and $l$. Coefficients $u_{d_{k}^{*} i}$ and $u_{\tilde{t}_{k l} i}$ are determined by the genotypes of the $k$ th and $l$ th QTL (marker) for the $i$ th RIL line (Table 1).

Models (3) and (6) were working models for our QTL mapping approach in the RIL-based NCIII design under the $F_{\infty}$ metric model. Model (3) can be used to estimate the augmented additive $\left(a_{k}^{*}\right)$ and epistatic $\left(\vec{i}_{k l}\right)$ effects, and model (6) can be used to estimate the augmented dominance $\left(d_{k}^{*}\right)$ and epistatic $\left(\tilde{i}_{k l}\right)$ effects. (ii) Statistical genetic models for mapping QTL in the RIL-based NCIII design under the $\mathrm{F}_{2}$ metric model. The statistical genetic models for mapping QTL with RIL-based NCIII $Z_{1 i}$ and $Z_{2 i}$ data under the $F_{2}$ metric model have the same forms as those under the $\mathrm{F}_{\infty}$ metric model [Models (3) and (6)]. The detailed derivation is described in the Supplemental Information B. The comparisons of model parameter components under the $\mathrm{F}_{\infty}$ and $\mathrm{F}_{2}$ metric model are given in Table 2 .

\subsection{Genetic parameter estimation}

Models (3) and (6) can be uniformly written as

$$
y_{i}=\mu+\sum_{k=1}^{q} x_{k i} g_{k}+\sum_{k=1}^{q-1} \sum_{l=k+1}^{q} x_{k l i} i_{k l}+e_{i}=\mu+\sum_{j=1}^{p} z_{j i} \gamma_{j}+e_{i},
$$

where $\mu$ is the model mean; $g_{k}$ is the augmented main effect of the $k$ th QTL; $i_{k l}$ is the augmented epistatic effect between the $k$ th and $l$ th QTL; $p=\frac{1}{2} q(q+1)$ is the total number of genetic effects, including the augmented main and epistatic effects; and $e_{i} \sim N\left(0, \sigma^{2}\right)$ is the residual error.

The true number of QTL $(q)$ is an import parameter in model (7) and usually determined by two alternative strategies, i.e. model selection and shrinkage estimation. The latter includes the fully Bayesian [37], the penalized maximum likelihood [38,39], least absolute shrinkage and selection operator, and empirical Bayes (E-Bayes) [32,40]. The fully Bayesian method should be optimal in theory because it captures the most information from the data, but it is computationally intensive; the penalized maximum likelihood is an approximation to the fully Bayesian approach, while its main advantages over other methods are reflected by its simplicity and fast speed; least absolute shrinkage and selection operator is a good approach but the shrinkage is too weak for spurious effects and too strong for large effects; and the E-Bayes provides the optimal estimates of variance components, because shrinkage is very selective, with large effects subject to virtually no shrinkage while small effects are shrunk to zero [40]. Simulation studies have shown that

\begin{tabular}{|c|c|c|c|c|c|c|c|}
\hline \multirow{2}{*}{ Data } & \multirow{2}{*}{ Model } & \multicolumn{3}{|c|}{$\mathrm{F}_{\infty}$ metric model } & \multicolumn{3}{|c|}{$\mathrm{F}_{2}$ metric model } \\
\hline & & Model mean & Main effect & Epistatic effect & Model mean & Main effect & Epistatic effect \\
\hline$Z_{1}$ & (3) & $\mu_{Z_{1}}=2 \mu+\sum_{k=1}^{q} d_{k}$ & $a_{k}^{*}=a_{k}+\frac{1}{2} \sum_{l \neq k}^{q}\left(i_{a_{k} d_{l}}-i_{d_{k} a_{l}}\right)$ & $\vec{i}_{k l}=i_{a_{k} a_{l}}+i_{d_{k} d_{l}}$ & $\mu_{Z_{1}}=2 \mu-\frac{1}{2} \sum_{k=1}^{q-1} \sum_{l=k+1}^{q} i_{d_{k} d_{l}}$ & $a_{k}^{*}=a_{k}-\frac{1}{2} \sum_{l \neq k}^{q} i_{d_{k} a_{l}}$ & $\vec{i}_{k l}=i_{a_{k} a_{l}}+i_{d_{k} d_{l}}$ \\
\hline$Z_{2}$ & (6) & $\mu_{Z_{2}}=\sum_{k=1}^{q} a_{k}$ & $d_{k}^{*}=d_{k}-\frac{1}{2} \sum_{l \neq k}^{q}\left(i_{a_{k} a_{l}}-i_{d_{k} d_{l}}\right)$ & $\tilde{i}_{k l}=i_{a_{k} d_{l}}+i_{d_{k} a_{l}}$ & $\mu_{Z_{2}}=\sum_{k=1}^{q} a_{k}$ & $d_{k}^{*}=d_{k}-\frac{1}{2} \sum_{l \neq k}^{q} i_{a_{k} a_{l}}$ & $\tilde{i}_{k l}=i_{a_{k} d_{l}}+i_{d_{k} a_{l}}$ \\
\hline
\end{tabular}
the E-Bayes is the best among the above approaches in terms of small mean squares error [40]. Thus, we adopted the E-Bayes method of $\mathrm{Xu}$ [32] for the estimation of parameters

Table 2 Model parameter components for $Z_{1 i}$ and $Z_{2 i}$ in the RIL-based NCIII under the $\mathrm{F}_{\infty}$ and $\mathrm{F}_{2}$ metric models 
in the above model. The approach assumes that there is one QTL standing on each marker throughout the genome and shrinks the genetic effects of all "nonsignificant" QTL toward zero. For the technical details of the method, refer to the original study by $\mathrm{Xu}$ [32] and its application in our research [41].

\subsection{Likelihood ratio test}

The usual likelihood ratio test (LRT) cannot be carried out with the E-Bayes method owing to an oversaturated epistatic genetic model. We proposed the following two-stage selection process to screen the markers [38]. In the first stage, all markers with $t_{j}=\left|\hat{\gamma}_{j}\right| / \hat{\sigma}_{j}>2.0$, where $\hat{\sigma}_{j}$ estimate of standard deviation for normal prior $\gamma_{j} \sim N\left(0, \sigma_{j}^{2}\right)$, are picked up. In the second stage, the epistatic genetic model is modified so that only effects past the first round of selection are included in the model. Owing to the smaller dimensionality of the reduced model, we can use the maximum likelihood method to re-analyze the data and perform the LRT [38]. The test statistic is

$$
L R_{j}=-2\left[L\left(\boldsymbol{\theta}_{-j}\right)-L(\boldsymbol{\theta})\right],
$$

where $\boldsymbol{\theta}$ is the parameters vector in the statistical genetic model in the second stage analysis of model (7); $\boldsymbol{\theta}_{-j}$ is the parameters vector in $\boldsymbol{\theta}$ excluding the currently tested genetic effect $\gamma_{j} ; L(\boldsymbol{\theta})$ and $L\left(\boldsymbol{\theta}_{-j}\right)$ are the $\log$ maximum likelihood function for $\boldsymbol{\theta}$ and $\boldsymbol{\theta}_{-j}$, respectively.

\section{Monte Carlo simulation studies}

The purpose of the simulation experiment was: (1) to evaluate the statistical performance of the proposed approach, and (2) to compare the RIL-based NCIII design with the $\mathrm{F}_{2}$-based NCIII design [41].

The simulated genome was $1000.0 \mathrm{cM}$ in total length and covered by 210 markers (10 chromosomes, each covered with twenty-one $5.0 \mathrm{cM}$ equally spaced markers). Ten QTL were assumed, of which four $\left(\mathrm{QTL}_{1}, \mathrm{QTL}_{2}, \mathrm{QTL}_{3}\right.$ and $\left.\mathrm{QTL}_{6}\right)$ had only main effects, and the other six had pairwise epistatic effects $\left(\mathrm{QTL}_{4}\right.$ and $\mathrm{QTL}_{7}, \mathrm{QTL}_{5}$ and $\mathrm{QTL}_{8}$, and QTL and QTL $_{10}$ interacted each other, respectively) (Tables 3-6 and S5-S8). Two independent QTL $\left(\mathrm{QTL}_{1}\right.$ and $\left.\mathrm{QTL}_{2}\right)$ and two interactive QTL (QTL 9 and $\mathrm{QTL}_{10}$ ) were in linkage disequilibrium, respectively, and the other six QTL were located on different linkage group. The pure main and epistatic effects under both the $\mathrm{F}_{\infty}$ and the $\mathrm{F}_{2}$ metric models were set as the same. The environmental variance was set at 6.0. Under the $\mathrm{F}_{\infty}$ ( or $\mathrm{F}_{2}$ ) metric model, the heritabilities for the ten main QTL varied from 0.00 to $12.69 \%$ (0.00 to $13.55 \%)$ in the $\mathrm{L}_{1}$ population and from 0.00 to $5.44 \%(0.00$ to $6.07 \%$ ) in the $\mathrm{L}_{2}$ population; those for the three epistatic QTL were $0.51,7.31$, and $0.00 \%(0.14,4.10$ and $0.00 \%)$ in the $\mathrm{L}_{1}$ population, and $15.70,7.83$, and $4.98 \%$ (23.77, 2.18 and $9.47 \%$ ) in the $\mathrm{L}_{2}$ population; the total heritabilities explained by all the main and epistatic QTL were 51.28 and $42.02 \%$ (47.95 and $35.26 \%$ ) in the above two populations, respectively (Table $\mathrm{S} 4$ ). Judged by pure main effects, $\mathrm{QTL}_{3}$ and $\mathrm{QTL}_{10}$ were additive; $\mathrm{QTL}_{1}$ and $\mathrm{QTL}_{4}$ were partially dominant; $\mathrm{QTL}_{2}$ was completely dominant; $\mathrm{QTL}_{6}, \mathrm{QTL}_{7}$ and $\mathrm{QTL}_{9}$ were overdominant; and $\mathrm{QTL}_{5}$ and $\mathrm{QTL}_{8}$ had no pure main effects (Tables 3, 5, S5 and S7). While judged by augmented main effects under the $\mathrm{F}_{\infty}$ metric model, $\mathrm{QTL}_{5}$ and $\mathrm{QTL}_{8}$ turned to complete dominance; the other eight QTL had the same types as the original ones, although degrees of dominance for some QTL $\left(\mathrm{QTL}_{4}\right.$ and $\left.\mathrm{QTL}_{7}\right)$ changed (Tables 3 and 5). However, when judged by augmented main effects under the $\mathrm{F}_{2}$ metric model, $\mathrm{QTL}_{5}$ and $\mathrm{QTL}_{8}$ became complete dominance and overdominance, respectively; $\mathrm{QTL}_{4}$ changed from partial dominance to overdominance, and on the contrary, $\mathrm{QTL}_{7}$ changed from overdominance to partial dominance; $\mathrm{QTL}_{9}$ and $\mathrm{QTL}_{10}$ turned to complete dominance from overdominance and additive, respectively; and the other four independent QTL had the same types as the original ones (Tables S5 and S7). The alteration of dominant mode for those interactive QTL was due to the existence of epistatic effects. The sample size $(n)$, the family number of the RIL population, was set at two levels: 300 and 500. The replication number $(\mathrm{m})$ for each NCIII family was set at 5 and 10 . Each treatment was replicated 200 times. With $Z_{1}$ or $Z_{2} 210$ augmented additive or dominance effects ( $a_{k}^{*}$ or $\left.d_{k}^{*}\right)$ and $\frac{1}{2} \times 210 \times(210-1)=21945$ augmented epistatic effects $\left(\vec{i}_{k l}\right.$ or $\tilde{i}_{k l}$ ) were included in the same one model and estimated simultaneously by empirical Bayes. All effects with $t_{j}=\left|\hat{b}_{j}\right| / \hat{\sigma}_{j}>2.0$ were picked up. All the screened effects were re-estimated by the maximum likelihood method and tested by LRT. For each simulated QTL, we counted the samples in which the LOD statistic was greater than 3.0 and the identified QTL was within $20.0 \mathrm{cM}$ of the simulated QTL. The estimate for QTL parameter was the average of the corresponding estimates in the counted samples. The ratio of the number of such samples to the total number of replicates represented the empirical power of this QTL.

To achieve the first objective of the simulation experiment, all the samples were analyzed by the proposed approach in this study. The mapping results under the $\mathrm{F}_{\infty}$ metric model were presented in Tables 3 and 4 for $n=300$ and Tables 5 and 6 for $n=500$. The results show that all the augmented main and epistatic effects were rightly and unbiasedly estimated (Tables 3-6). All of the main-effect QTL were identified with a high statistical power and precision of estimated effects and positions. The statistical powers in the detection of augmented additive and dominant effects $\left(a_{k}^{*}\right.$ and $d_{k}^{*}$ ) and precisions of its effects were comparable when they had equivalent size (Tables 3 and 5). The epi- 
static QTL $\left(\mathrm{QTL}_{4} \times \mathrm{QTL}_{7}\right.$ and $\left.\mathrm{QTL}_{9} \times \mathrm{QTL}_{10}\right)$ were also very well mapped for all simulated situations when the augmented epistatic effects consisted of two pure epistatic effects in the same sign (Tables 4 and 6). The power for uncovering epistatic QTL $\left(\mathrm{QTL}_{5} \times \mathrm{QTL}_{8}\right)$, of which the augmented epistatic effects consisted of two pure epistatic effects in opposite directions, were very low (25.0\% for $\vec{i}_{58}$ and $28.5 \%$ for $\tilde{i}_{58}$ ) when both sample size and replication number were at low levels $(n=300, m=5)$; increased to satisfactory values $(63.0 \%$ to $69.5 \%)$ when either sample size or replication number was at high level $[(n=300$ and $m=10)$ or $(n=500$ and $m=5)$ ]; and rose to high quantities $(96.0 \%$ and $97.0 \%$ ) when both sample size and replication number were at a high level ( $n=500$ and $m=10)$ (Tables 4 and 6). The statistical powers for detection of the two types of augmented epistatic effects $\left(\vec{i}_{k l}\right.$ and $\left.\tilde{i}_{k l}\right)$ and precisions of its effects were also comparable when they had equal size (Tables 4 and 6). The detection of the augmented epistatic effects $\left(\vec{i}_{k l}\right.$ and $\left.\tilde{i}_{k l}\right)$ was relatively more difficult than that of the augmented main effects ( $a_{k}^{*}$ and $d_{k}^{*}$ ), especially when they had small effects (Tables 3-6). The time costs were about 2.30 and $4.90 \mathrm{~h}$ per sample on our computer (CPU: Intel ${ }^{\circledR}$ Core $^{\mathrm{TM}} 2$ DUO 3.0G, Memory: 2.0G) for sample size 300 and 500, respectively.

The results under the $\mathrm{F}_{2}$ metric model are listed in Tables S5 and S6 for $n=300$ and Tables S7 and S8 for $n=500$. The general conclusions were similar to those under the $\mathrm{F}_{\infty}$ metric model, although the augmented main effects $\left(a_{k}^{*}\right.$ and $d_{k}^{*}$ ) with the interactive QTL under the two models were different. In addition, the false-positive rates (FPR), the ratio of the number of false-positive effects to the total number of zero effects considered in models (3) and (6), were low $\left(1 \times 10^{-4}\right.$ to $2 \times 10^{-4}$ mostly, Table S9).

To achieve the second objective of the simulation experiment, the above results were compared with results in $\mathrm{He}$ and Zhang [41]. In He and Zhang [41], the parameter settings in the $\mathrm{F}_{2}$-based NCIII design were similar to those in this study. The results showed that the RIL-based NCIII design was superior to the $\mathrm{F}_{2}$-based NCIII design for mapping heterotic QTL. Although the augmented main effects $\left(a^{*}\right.$ and $\left.d^{*}\right)$ were all unbiasedly estimated with satisfactory powers and the augmented epistatic effects $(\vec{i}$ and $\tilde{i})$ between QTL $_{4}$ and QTL $_{7}$, which consisted of two epistatic effects with the same sign, were also well mapped. The augmented epistatic effects $\left(\vec{i}_{58}\right.$ and $\tilde{i}_{58}$ between QTL and $\mathrm{QTL}_{8}$ ), which consisted of two epistatic effects of equal strength in opposite directions, were very poorly mapped, even though both sample size and replication number were at high levels $(n=500$ and $m=10)$, i.e., the powers in the detection of $\vec{i}_{58}$ and $\tilde{i}_{58}$ in the $\mathrm{F}_{2}$-based NCIII design were 0.50 and $2.00 \%$ under the $\mathrm{F}_{\infty}$ metric model, and 0.50 and
$18.5 \%$ under the $\mathrm{F}_{2}$ metric model [41].

Assuming that a quantitative trait was controlled by only two QTL with digenic epistasis and arbitrary linkage, the genetic variances of $Z_{1 i}$ and $Z_{2 i}$ among the RIL-based NCIII families have the same expression under the $\mathrm{F}_{\infty}$ and $\mathrm{F}_{2}$ metric models when the symbols of augmented effects are used (Supplement Information A),

$$
\begin{aligned}
\sigma_{Z_{1}}^{2}(\mathrm{RIL})= & \left(a_{1}^{*}\right)^{2}+\left(a_{2}^{*}\right)^{2}+R(1-R){\overrightarrow{i_{12}}}^{2} \\
& +2(1-2 R) a_{1}^{*} a_{2}^{*} \stackrel{r=R=0.5}{=}\left(a_{1}^{*}\right)^{2}+\left(a_{2}^{*}\right)^{2}+\frac{1}{4} \vec{i}_{12}^{2}, \\
\sigma_{Z_{2}}^{2}(\mathrm{RIL})= & \left(d_{1}^{*}\right)^{2}+\left(d_{2}^{*}\right)^{2}+R(1-R) \tilde{i}_{12}^{2} \\
& +2(1-2 R) d_{1}^{*} d_{2}^{* r=R=0.5}=\left(d_{1}^{*}\right)^{2}+\left(d_{2}^{*}\right)^{2}+\frac{1}{4} \tilde{i}_{12}^{2},
\end{aligned}
$$

where $R=2 r /(1+2 r)$ and $r$ is the recombination fraction between the two loci. Similarly, the genetic variances of $Z_{1 i}$ and $Z_{2 i}$ among the $\mathrm{F}_{2}$-based NCIII families also have the same expression under the $\mathrm{F}_{\infty}$ and $\mathrm{F}_{2}$ metric models,

$$
\begin{aligned}
\sigma_{Z_{1}}^{2}\left(\mathrm{~F}_{2}\right)= & \frac{1}{2}\left(a_{1}^{*}\right)^{2}+\frac{1}{2}\left(a_{2}^{*}\right)^{2}+\frac{1}{4} r\left(2-5 r+4 r^{2}\right) \vec{i}_{12}^{2} \\
& +(1-2 r) a_{1}^{*} a_{2}^{*} \stackrel{r=0.5}{=} \frac{1}{2}\left(a_{1}^{*}\right)^{2}+\frac{1}{2}\left(a_{2}^{*}\right)^{2}+\frac{1}{16} \ddot{i}_{12}^{2}, \\
\sigma_{Z_{2}}^{2}\left(\mathrm{~F}_{2}\right)= & \frac{1}{2}\left(d_{1}^{*}\right)^{2}+\frac{1}{2}\left(d_{2}^{*}\right)^{2}+\frac{1}{4} r\left(2-5 r+4 r^{2}\right) \tilde{i}_{12}^{2} \\
& +(1-2 r) d_{1}^{*} d_{2}^{*} \stackrel{r=0.5}{=} \frac{1}{2}\left(d_{1}^{*}\right)^{2}+\frac{1}{2}\left(d_{2}^{*}\right)^{2}+\frac{1}{16} \tilde{i}_{12}^{2} .
\end{aligned}
$$

These variance formulae were helpful for explaining the above results.

\section{Discussion}

NCIII design is widely used to infer the genetic basis of heterosis for many economically important traits. However, previous studies have shown that a conclusion on the genetic basis of heterosis depends on a statistical approach adopted by $[8,16,17,36]$. Therefore, the development and choice of appropriate methodology for NCIII design are critical. In Stuber et al. [19] and Xiao et al. [22], the separate analysis on each backcross population actually estimated $d^{*}+a^{*}$ or $d^{*}-a^{*}[11,14,16]$. To overcome this issue, Cockerham and Zeng [11] separated the additive and dominance effects in their combined analysis. However, their single marker analysis method could not separate multiple linked gene effects and did not provide exact location of the QTL $[11,16]$. Based on this situation, Lu et al. [12] and Ledeaux et al. [28] developed the use of composite interval mapping [27] on each backcross separately and, after QTL are mapped in one or both backcrosses, $a^{*}$ and $d^{*}$ effects were estimated by a linear combination of the contrasts for each backcross according to the derivations of Cockerham and Zeng [11]. Although $a^{*}$ and $d^{*}$ effects can be indirectly estimated, the results of QTL mapping are still based on the analysis of each backcross separately in a similar way to 
that of Stuber et al. [19]. The extended CIM analysis on progeny mean values for pair means and pair differences, which correspond to contrasts $C_{1}$ and $C_{3}$ of Cockerham and Zeng [11], suggested by Melchinger et al. [14], not only can estimate $a^{*}$ and $d^{*}$ directly, but also can eliminate the influence of other linked QTL. However, the augmented epistatic effects $\left(\vec{i}=i_{a a}+i_{d d}\right.$ and $\left.\tilde{i}=i_{a d}+i_{d a}\right)$ are not included in the model of Melchinger et al. [14]. This avoidance would result in a larger residual variance, further a lower power of QTL detection and poorer precision (little larger in standard deviation) in estimated QTL effects and positions [41]. The two dimensional scanning methods, which consider both main and epistatic effects of pair QTL simultaneously with genetic background controlling, such as the mixed model approach of Wang et al. [23] and inclusive composite interval mapping (ICIM) of Li et al. [42], are alternative methods. However, the genetic design matrix should be reconstructed (Table 1) and the genetic parameter should be correctly explained [14-16,24,30,41]. Although the multiple interval mapping in Garcia et al. [16] can estimate the augmented main and epistatic effects simultaneously, it differs from our method in two aspects. First, Garcia et al. [16] adopt a model selection strategy, such as AIC criteria, to search the final model, whereas we fit all markers on the whole genome simultaneously. As a result, the latter may capture the best model more easily than the former. Second, the former uses orthogonal contrasts and the original observations, and we take advantage of linear regression and the two classical transformations.

The proposed approach is similar to He and Zhang's [41] method in spirit, but we extend He and Zhang's [41] method from an $\mathrm{F}_{2}$-based triple testcross (TTC) design to a RIL-based NCIII design. Models (3) and (6) can also be used for the NCIII design derived from other base populations, such as $\mathrm{DH}, \mathrm{F}_{2}, \mathrm{BC}$ and $\mathrm{F}_{2} \mathrm{Syn} 3$, after the genetic design matrix (Table 1) was appropriately reconstructed. For a quantitative trait determined by two unlinked QTL, the genetic variances among NCIII families on $Z_{1 i}\left[Z_{2 i}\right]$ are $\left(a_{1}^{*}\right)^{2}+\left(a_{2}^{*}\right)^{2}+\frac{1}{4} \vec{i}_{12}^{2} \quad\left[\left(d_{1}^{*}\right)^{2}+\left(d_{2}^{*}\right)^{2}+\frac{1}{4} \tilde{i}_{12}^{2}\right]$ and $\frac{1}{2}\left(a_{1}^{*}\right)^{2}+$ $\frac{1}{2}\left(a_{2}^{*}\right)^{2}+\frac{1}{16} \ddot{i}_{12}^{2}\left[\frac{1}{2}\left(d_{1}^{*}\right)^{2}+\frac{1}{2}\left(d_{2}^{*}\right)^{2}+\frac{1}{16} \tilde{i}_{12}^{2}\right]$ for RIL and $\mathrm{F}_{2}$, respectively. The coefficients for genetic variance components of the augmented main effects are two times larger for RIL than for $\mathrm{F}_{2}$; and it becomes four times when for augmented epistatic effects. Therefore, the RIL-based NCIII design is much more powerful in the detection of main, and especially epistatic QTL, than in the $\mathrm{F}_{2}$-based NCIII design. The conclusion is similar to that in Schön et al. [17] and is also confirmed by our simulated experiments. For example, in the detection of $\mathrm{QTL}_{5} \times \mathrm{QTL}_{8}$ interaction that consists of two epistatic effects in opposite directions, the power is satisfactory when either sample size or replication number is at a high level [ $(n=300$ and $m=10)$ or $(n=500$ and $m=5)]$ in the RIL-based NCIII design (Tables 4 and 6), and is ex- tremely low even when both sample size and replication number are at high levels $(n=500$ and $m=10)$ in the $\mathrm{F}_{2}$-based NCIII design. The result helps us to choose an optimal base population in their NCIII design.

The augmented additive effect $\left(a_{k}^{*}\right)$ equals to $a_{k}+$ $\frac{1}{2} \sum_{l \neq k}^{q}\left(i_{a_{k} d_{l}}-i_{d_{k} a_{l}}\right) \quad\left[a_{k}-\frac{1}{2} \sum_{l \neq k}^{q} i_{d_{k} a_{l}}\right]$ and the augmented dominant effect $\left(d_{k}^{*}\right)$ equals to $d_{k}-\frac{1}{2} \sum_{l \neq k}^{q}\left(i_{a_{k} a_{l}}-i_{d_{k} d_{l}}\right)$ $\left[d_{k}-\frac{1}{2} \sum_{l \neq k}^{q} i_{a_{k} a_{l}}\right]$ under the $\mathrm{F}_{\infty}\left[\mathrm{F}_{2}\right]$ metric model (Table 2). In these augmented main effects, the pure main effects ( $a_{k}$ and $d_{k}$ ) are confounded with pure epistatic interactions $\left(i_{a_{k} d_{l}}, i_{d_{k} a_{l}}, i_{a_{k} a_{l}}\right.$ and $\left.i_{d_{k} d_{l}}\right)$. However, the augmented additive and dominant effects just precisely measure the net contribution of the $k$-th QTL to parental difference and midparent heterosis; this is the advantage of the NCIII design [14]. However, the relative contribution of the pure main effects cannot be assessed independently from the sum of epistatic interactions of QTL $k$ with the genetic background, and this is a limitation of the NCIII design [14,36]. Likewise, the pure epistatic effects $\left(i_{a_{k} a_{l}}\right.$ and $i_{d_{k} d_{l}}, i_{a_{k} d_{l}}$ and $i_{d_{k} a_{l}}$ ) between QTL $k$ and $l$ are also confounded $\left(\vec{i}_{k l}=i_{a_{k} a_{l}}+i_{d_{k} d_{l}}\right.$ and $\left.\tilde{i}_{k l}=i_{a_{k} d_{l}}+i_{d_{k} a_{l}}\right)$ and cannot be individually estimated. If $i_{a_{k} a_{l}}$ and $i_{d_{k} d_{l}}\left(i_{a_{k} d_{l}}\right.$ and $\left.i_{d_{k} a_{l}}\right)$ have the same sign, the augmented epistatic effect $\vec{i}_{k l}\left(\tilde{i}_{k l}\right)$ will be inflated and can be more easily detected; if the two componential pure epistatic effects have like magnitude and opposite signs, the augmented epistatic effects will be weak and can be hardly detected [16](Tables 4, 6, S6 and S8 in this study). Fortunately, the RIL-based NCIII design has relatively high power for detecting $\vec{i}_{k l}$ and $\tilde{i}_{k l}$. By increasing sample size and/or replication number, we still have considerable power to indentify $\vec{i}_{k l}$ and $\tilde{i}_{k l}$, which consisted of two epistatic effects of equal strength in opposite directions (Tables 4, 6, S6 and S8). To dissect the augmented main and epistatic effects, a special mating design, such as the TTC design, will be needed. In the TTC design, the $\mathrm{F}_{1}$ is added as a third tester to produce the $L_{3 i}$ family and the third linear transformation $Z_{3 i}=\bar{L}_{1 i}+\bar{L}_{2 i}-2 \bar{L}_{3 i}$ is constructed. Under the $F_{2}$ metric model, Melchinger et al. [36] suggested that one- dimensional genome scans on $L_{3 i}$ and $Z_{3 i}$ can be adopted for separately estimating $a_{k}$ and $\sum_{l \neq k}^{q} i_{d_{k} a_{l}}$ in $a_{k}^{*}$; two-way ANOVA for marker interactions with $L_{3 i}$ can be used to acquire $i_{a_{k} a_{l}}$ and these $i_{a_{k} a_{l}}$ can be further used for dissection of heterotic-effect $d_{k}^{*}$. Under both the $\mathrm{F}_{\infty}$ and $\mathrm{F}_{2}$ metric models, He and Zhang [41] developed a two-step approach to completely dissect pure main and epistatic effects of QTL in the $F_{2}$-based TTC 


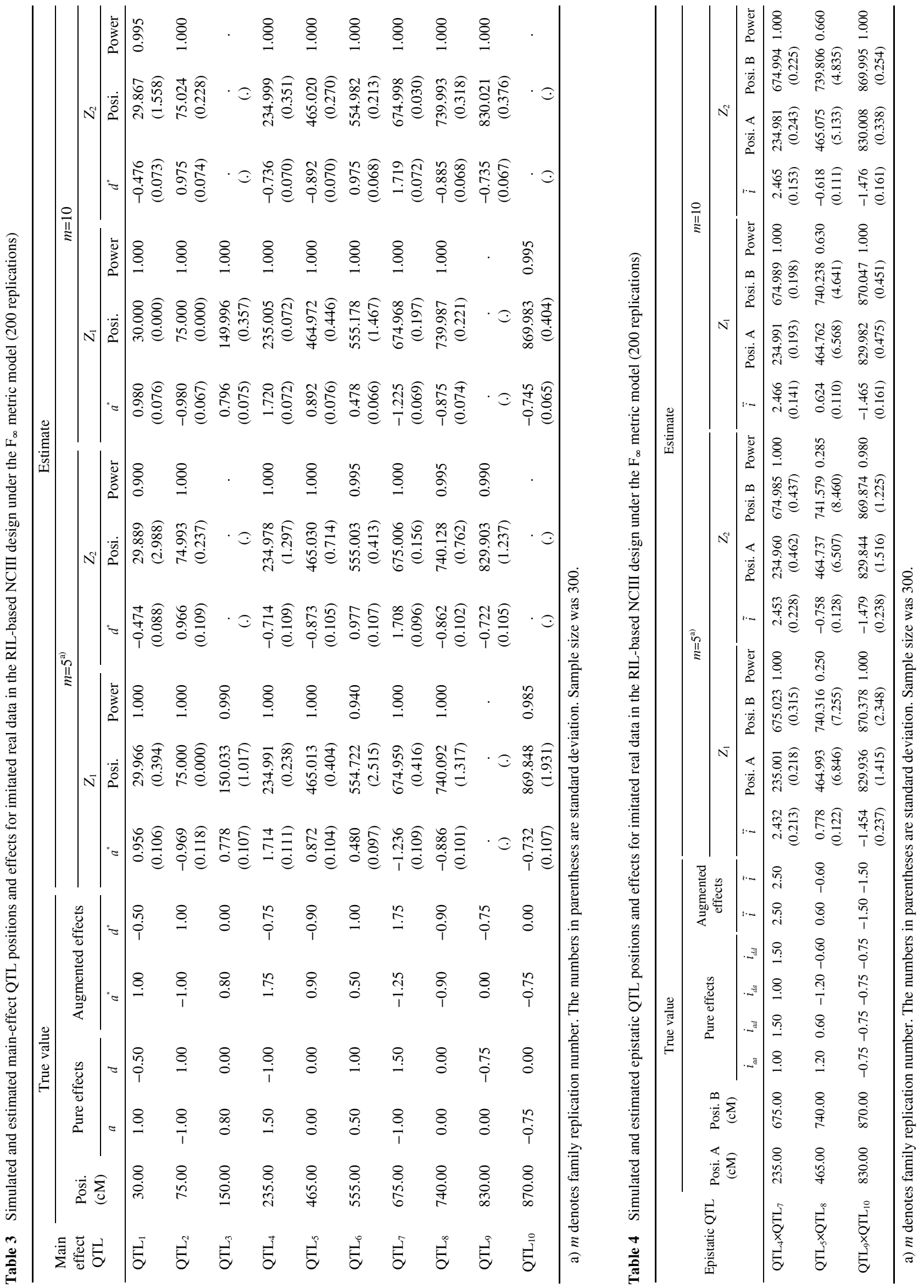

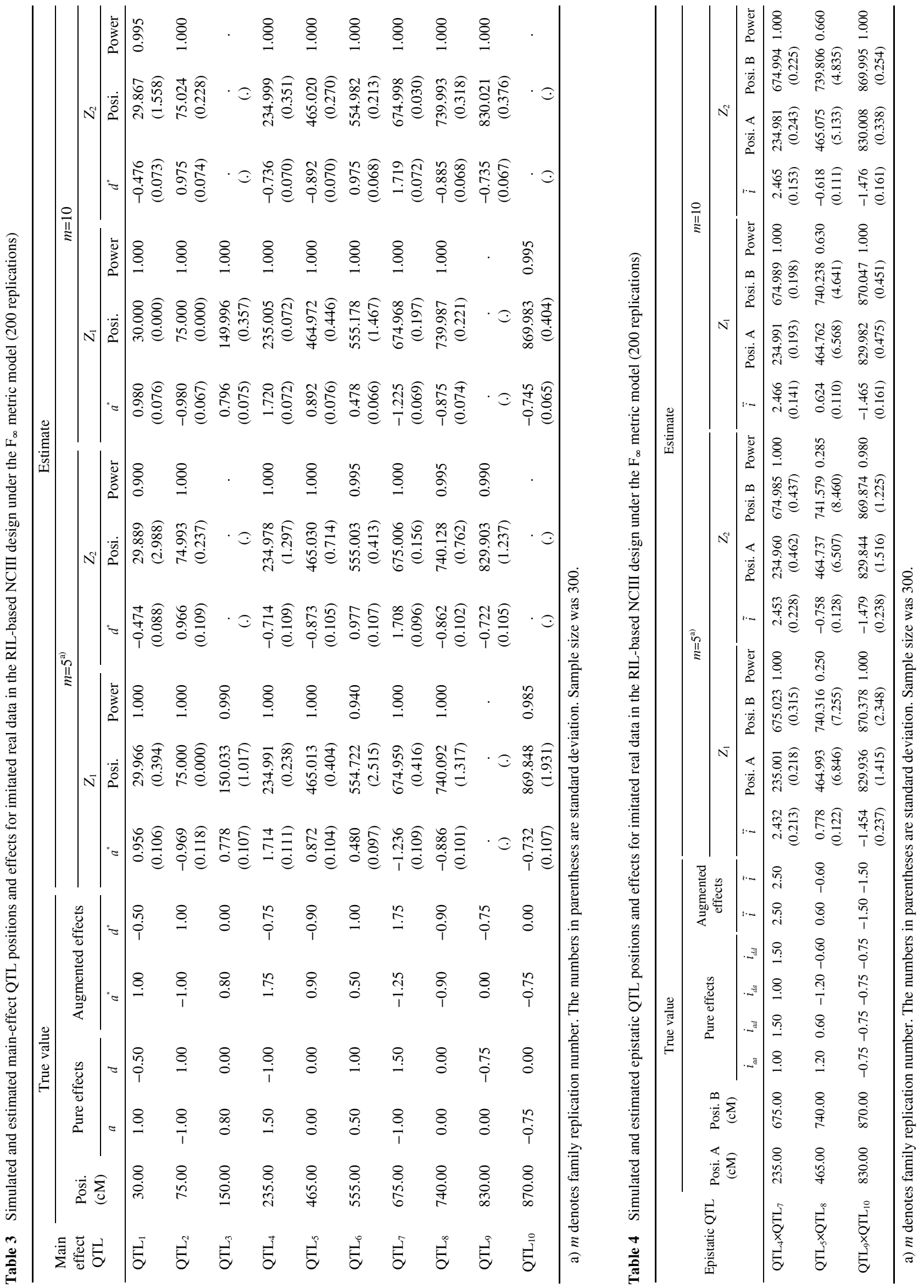

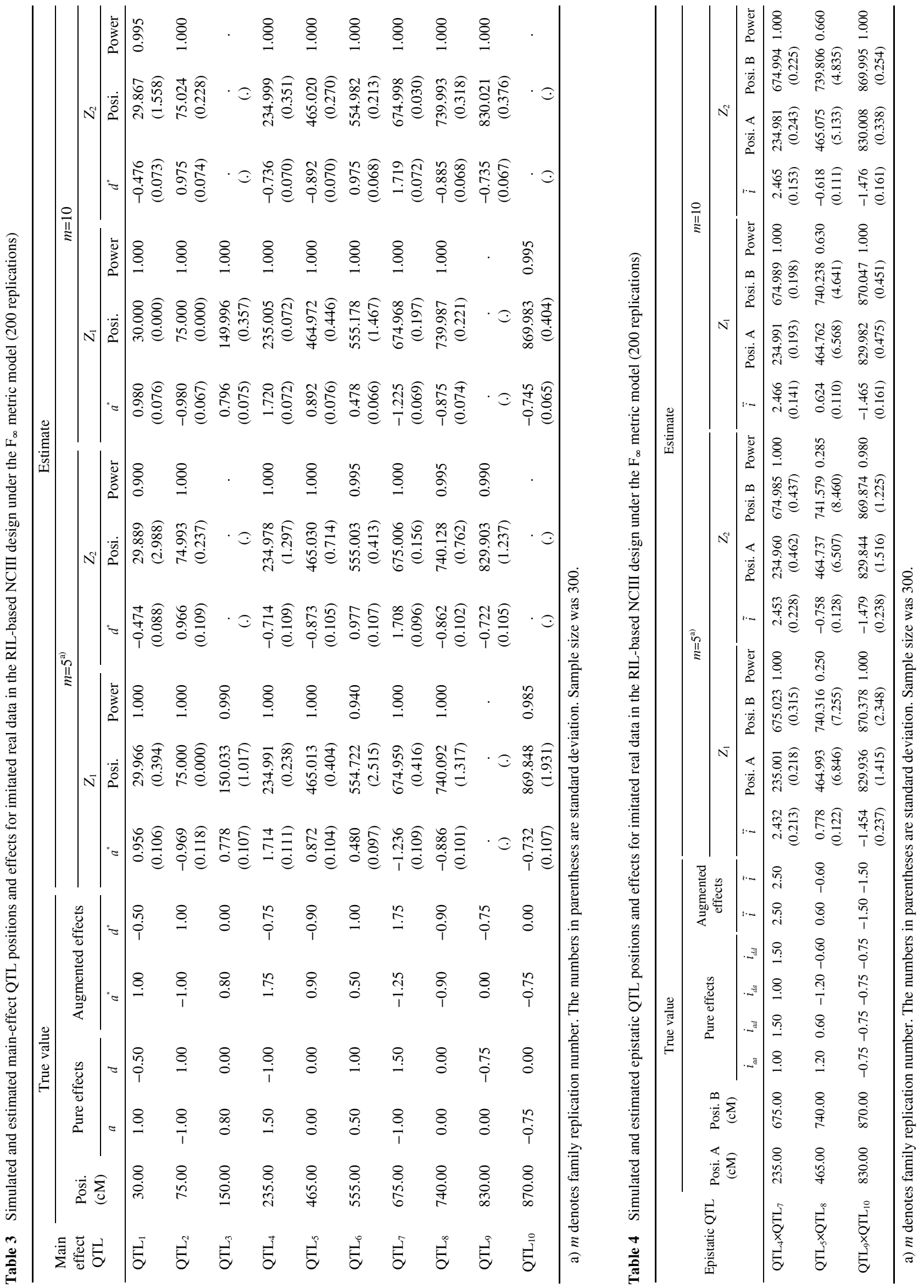

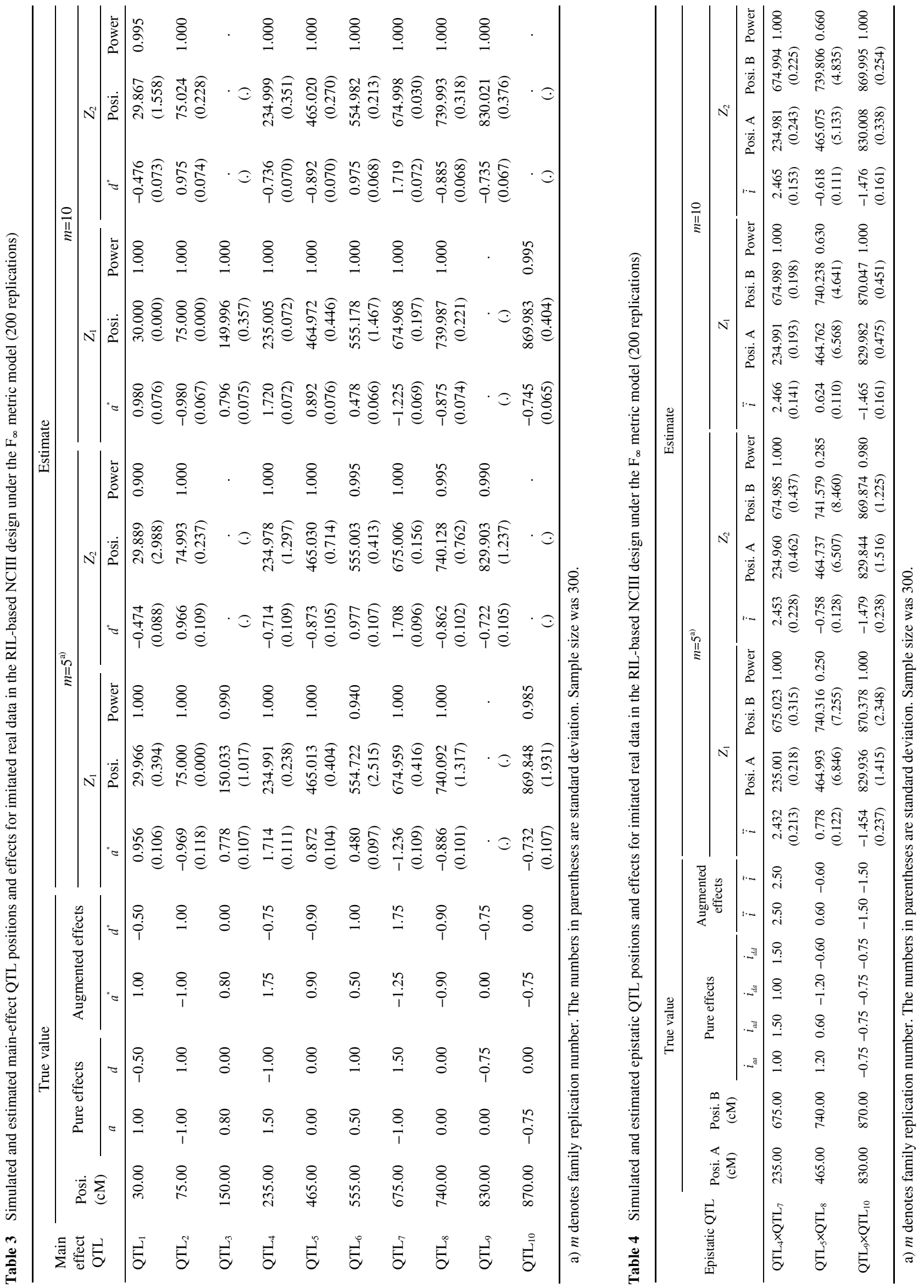

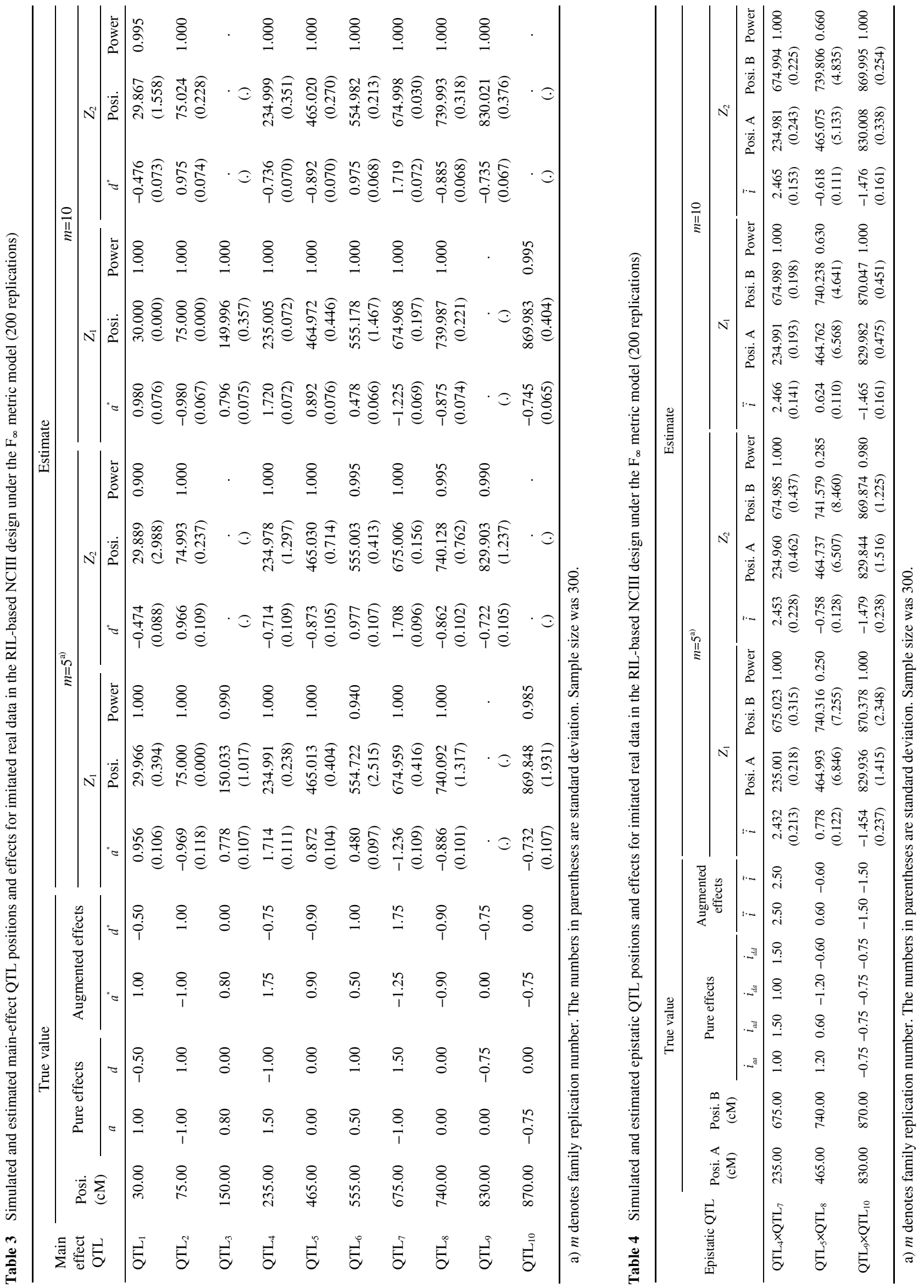

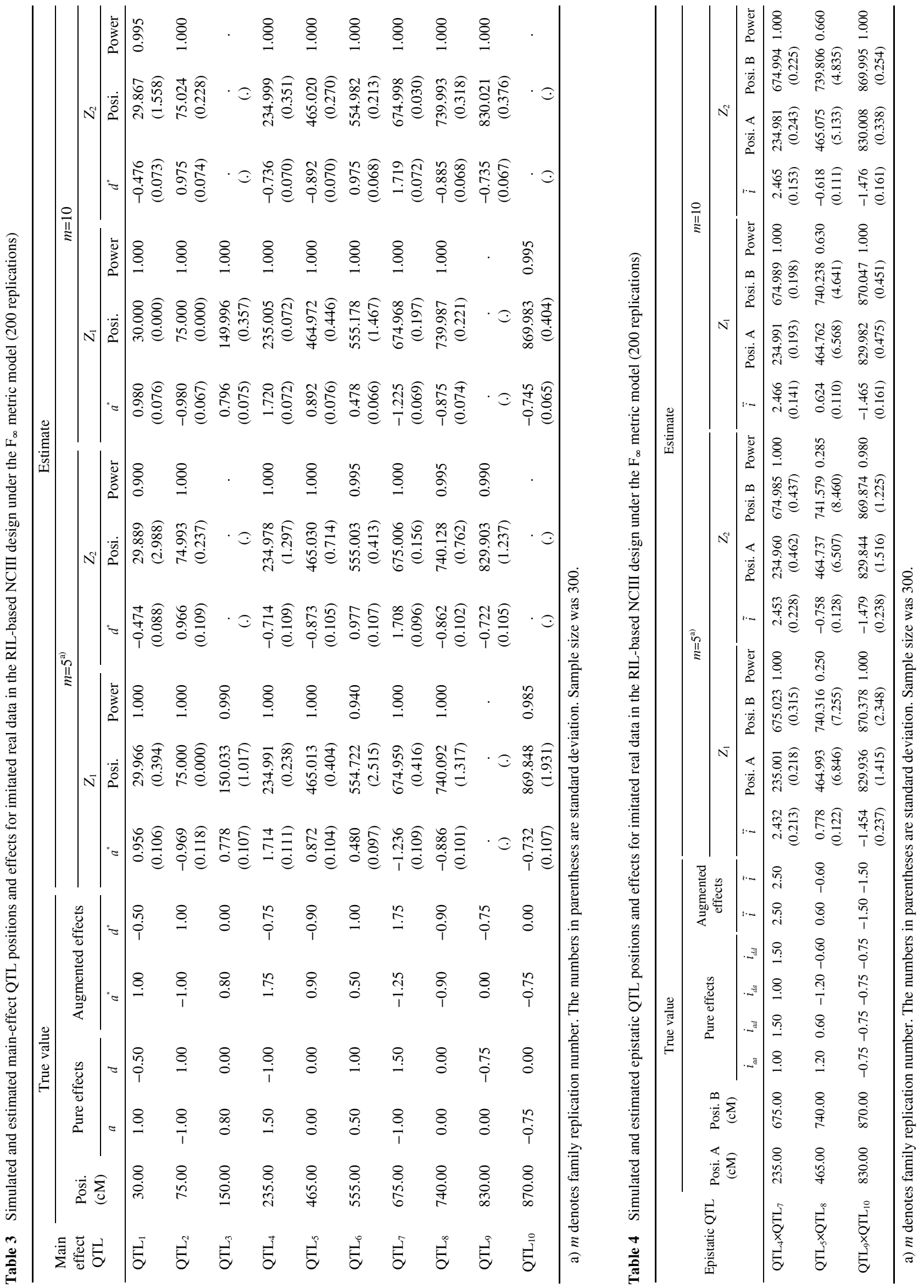

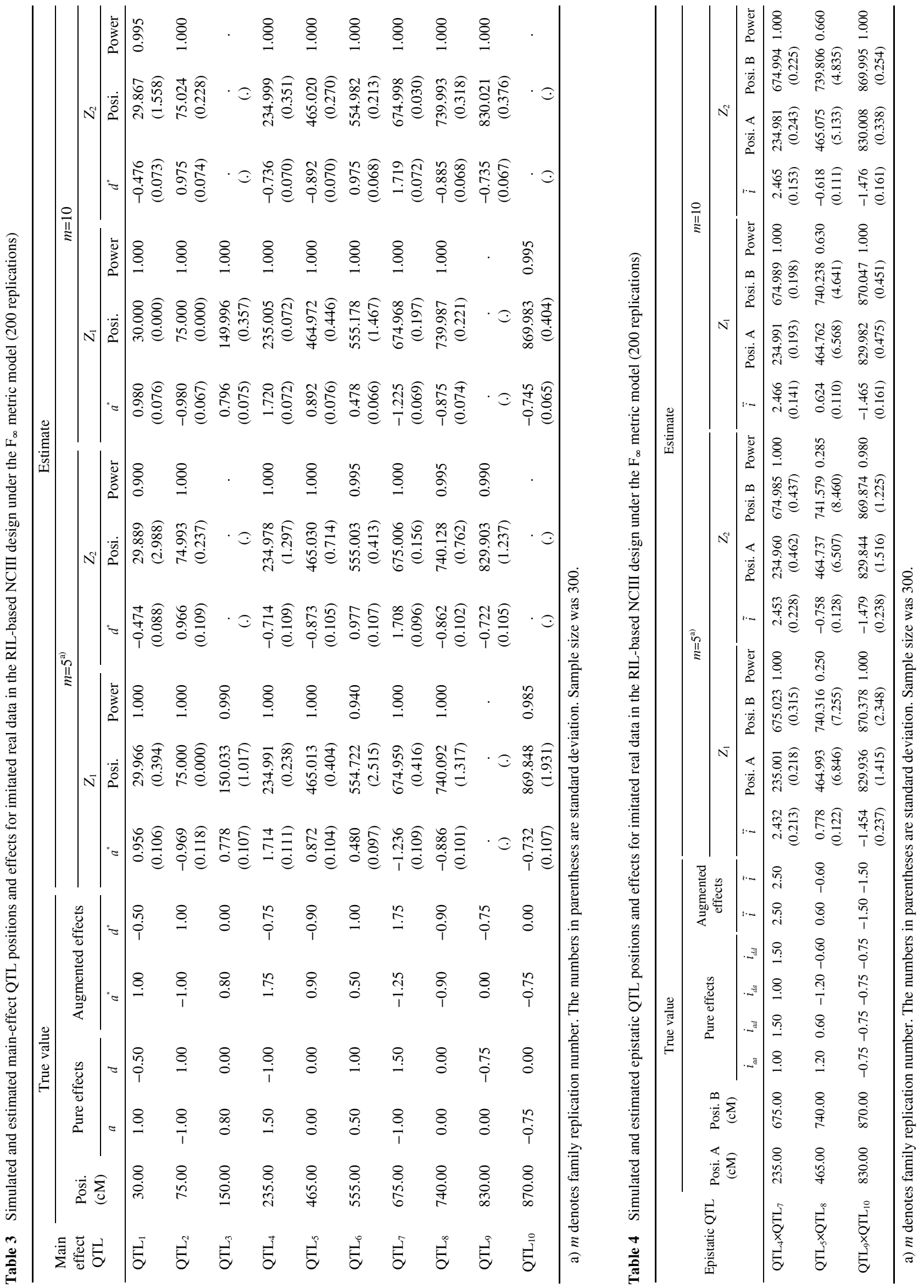

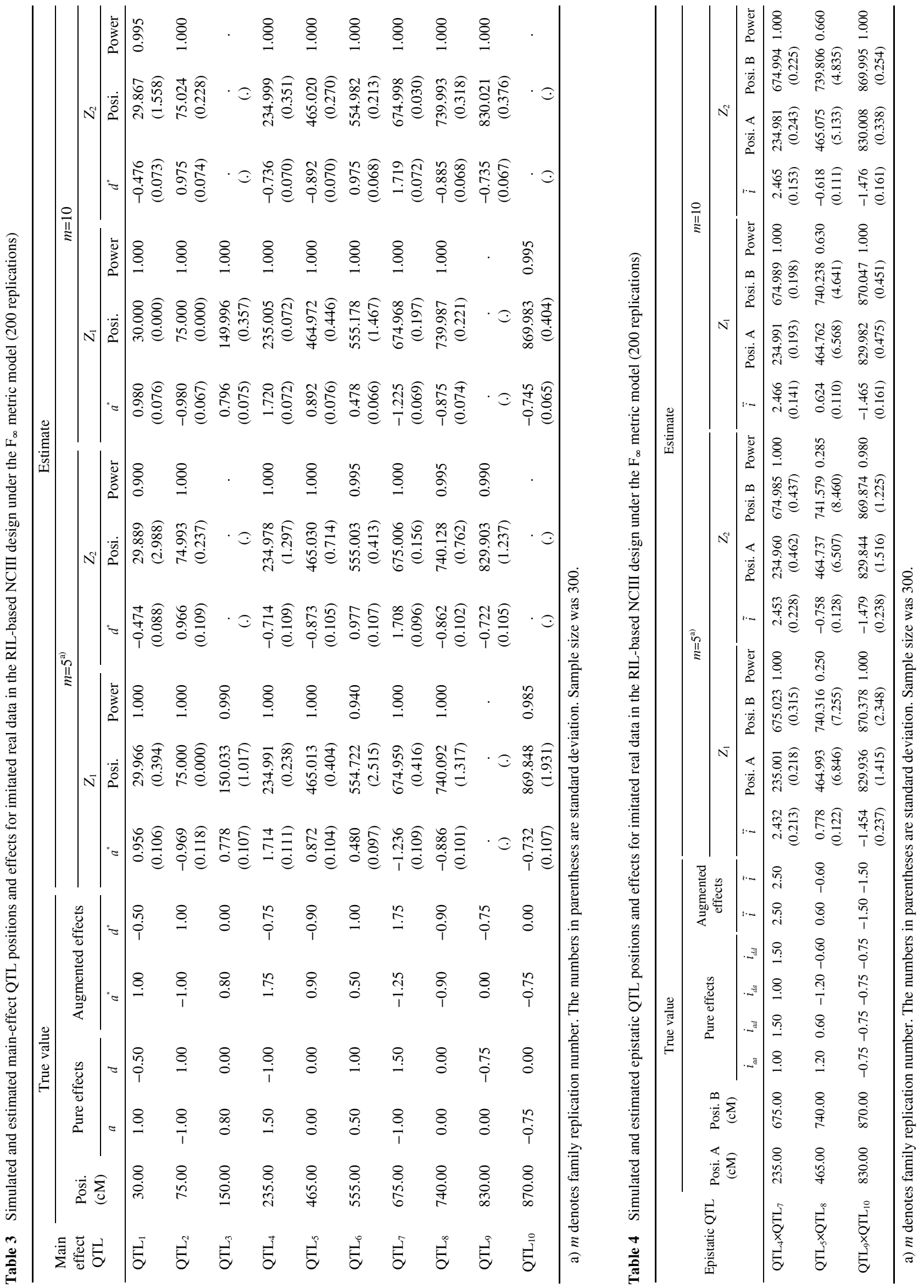

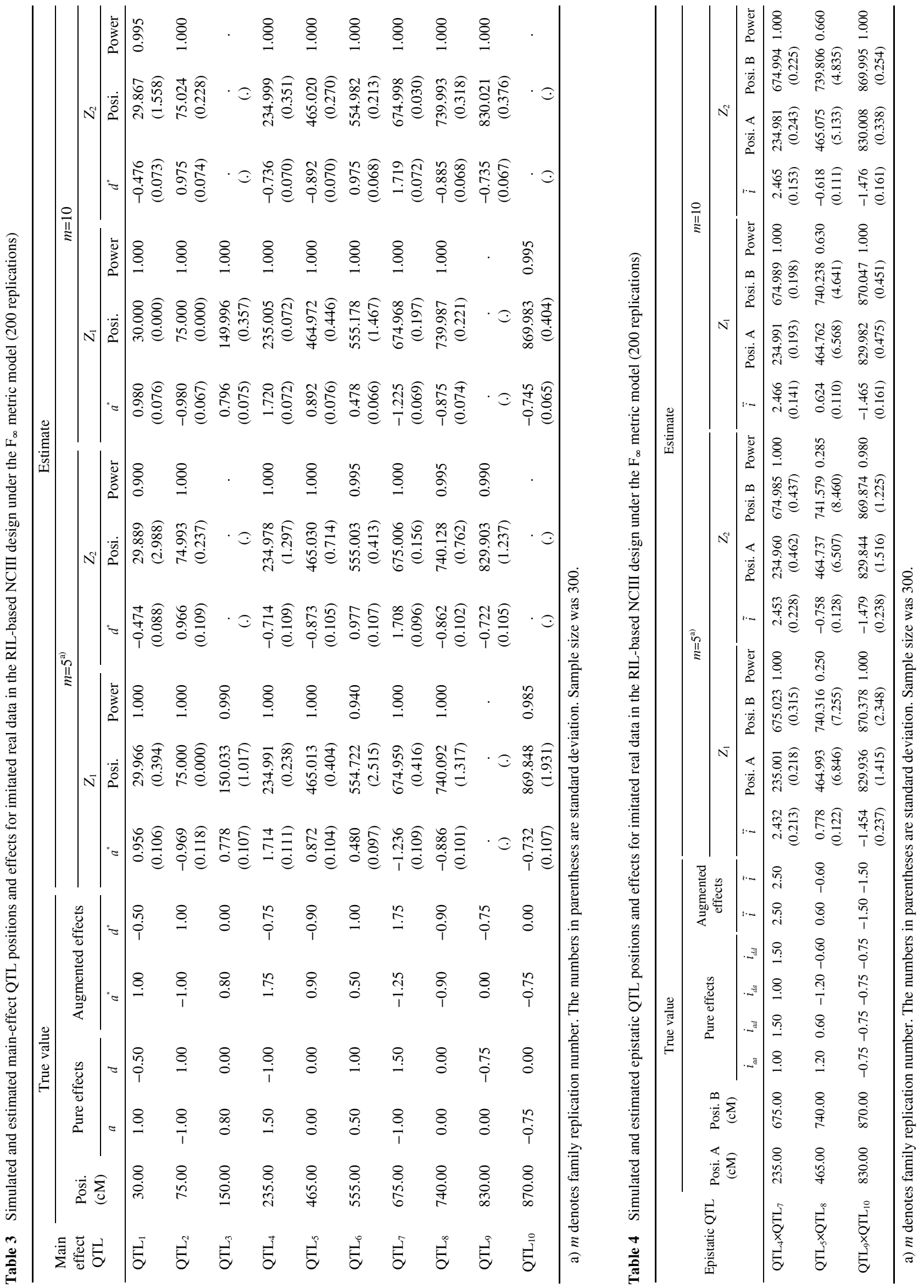

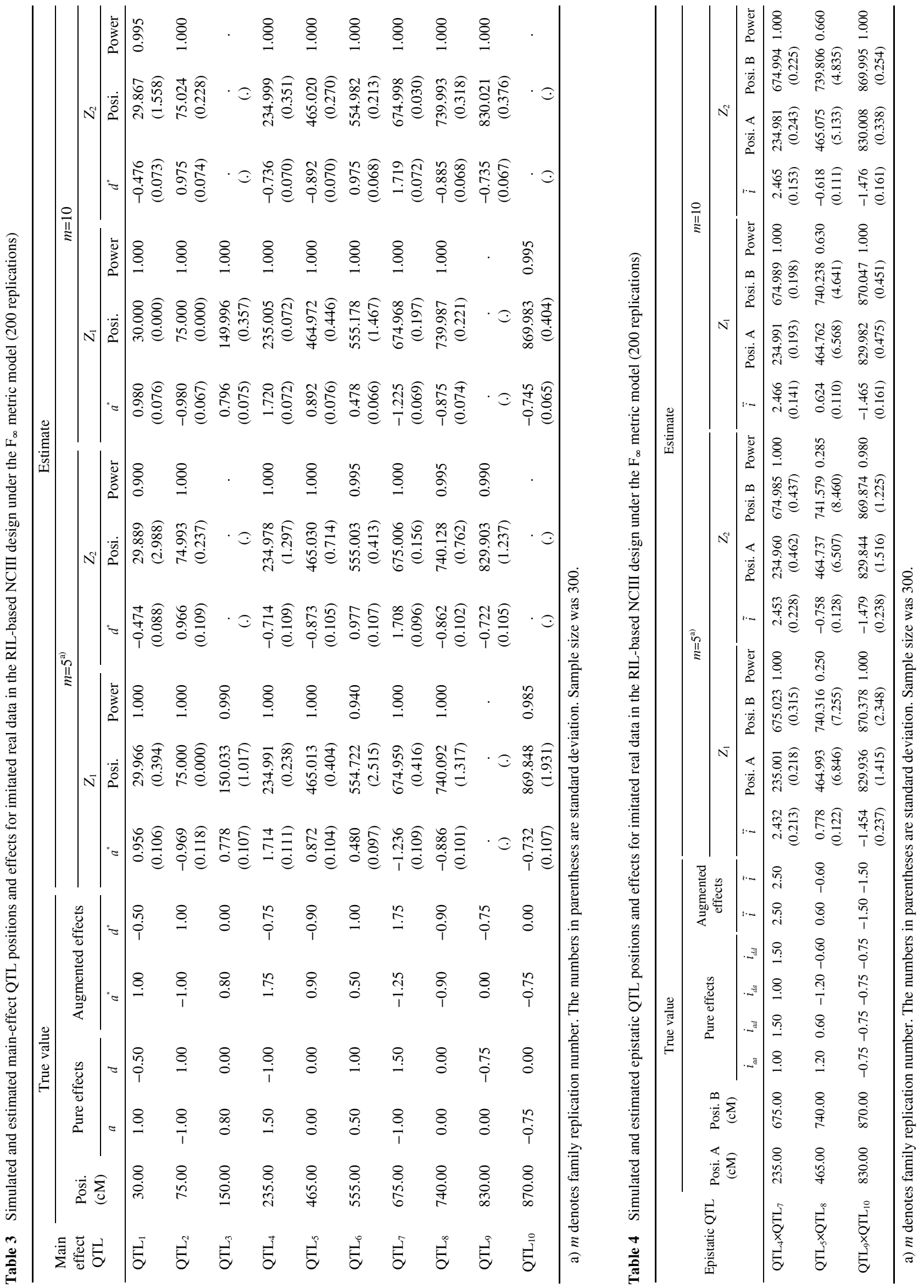

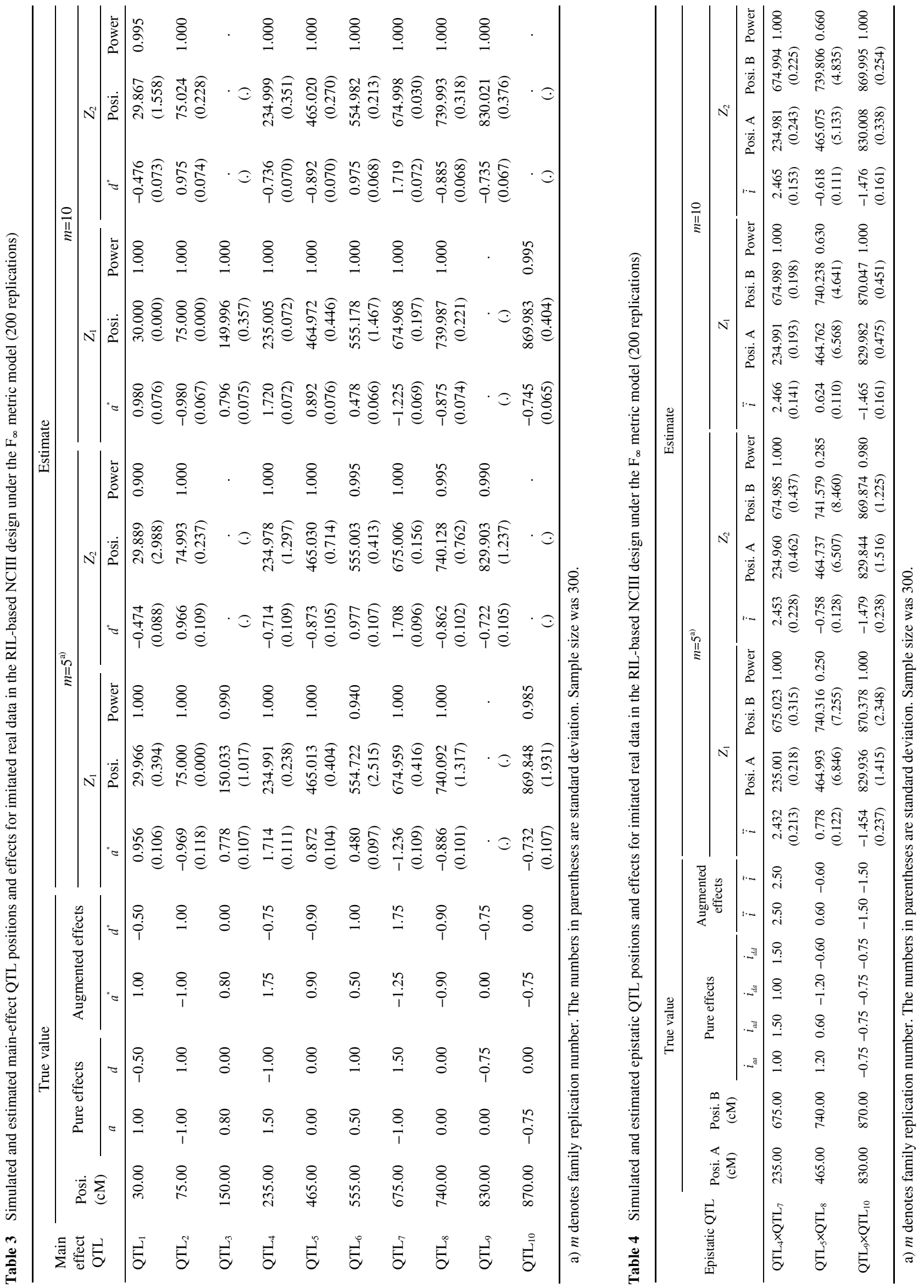

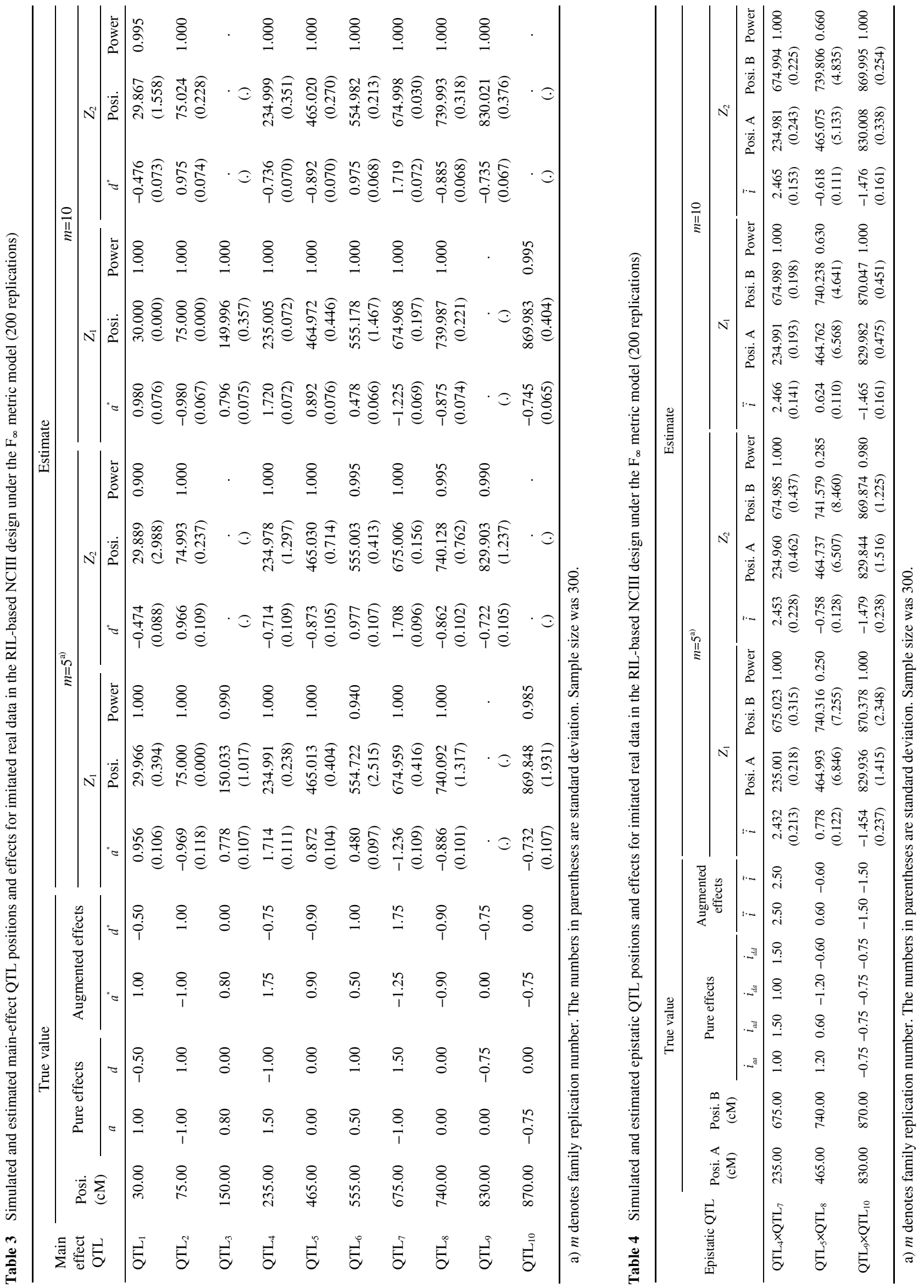

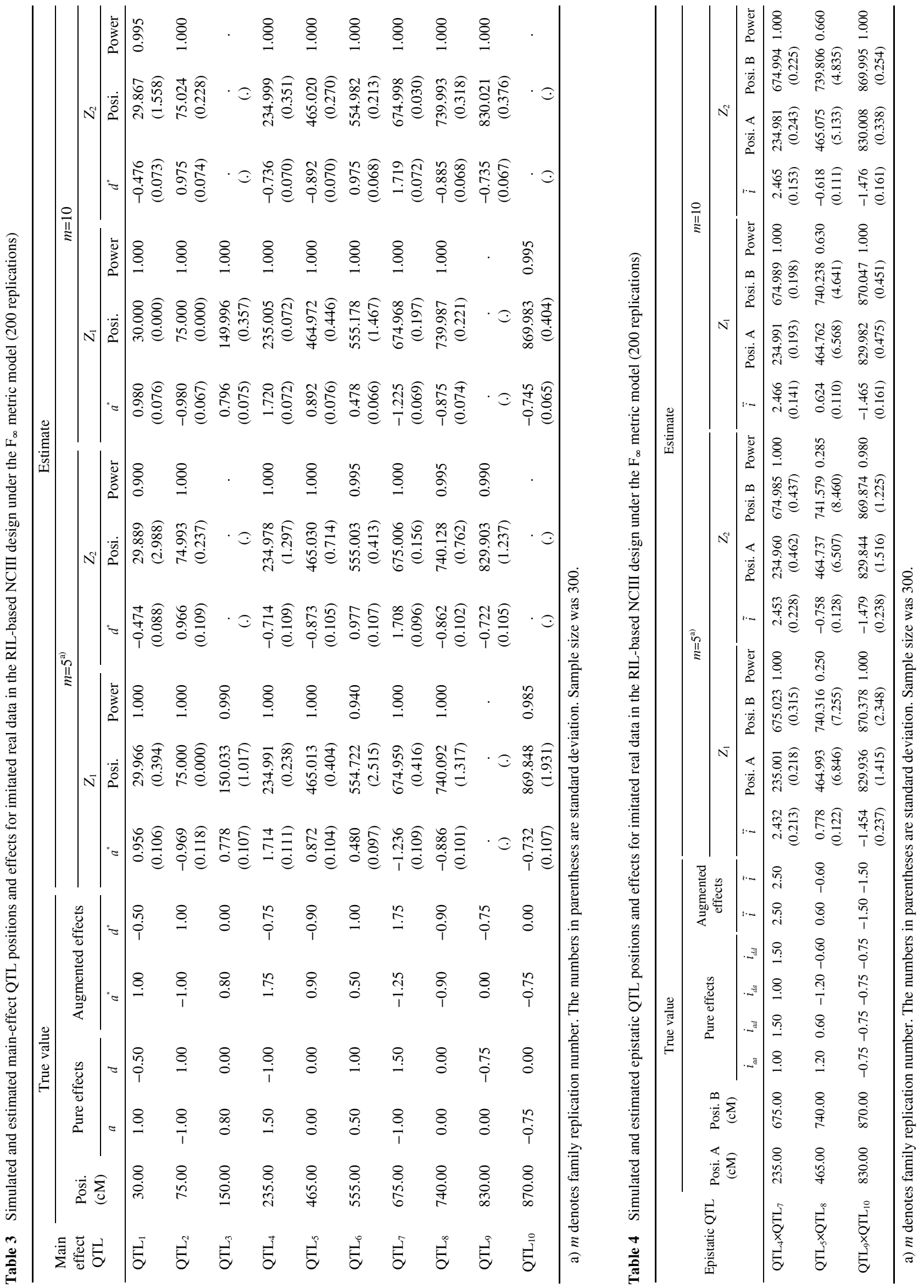

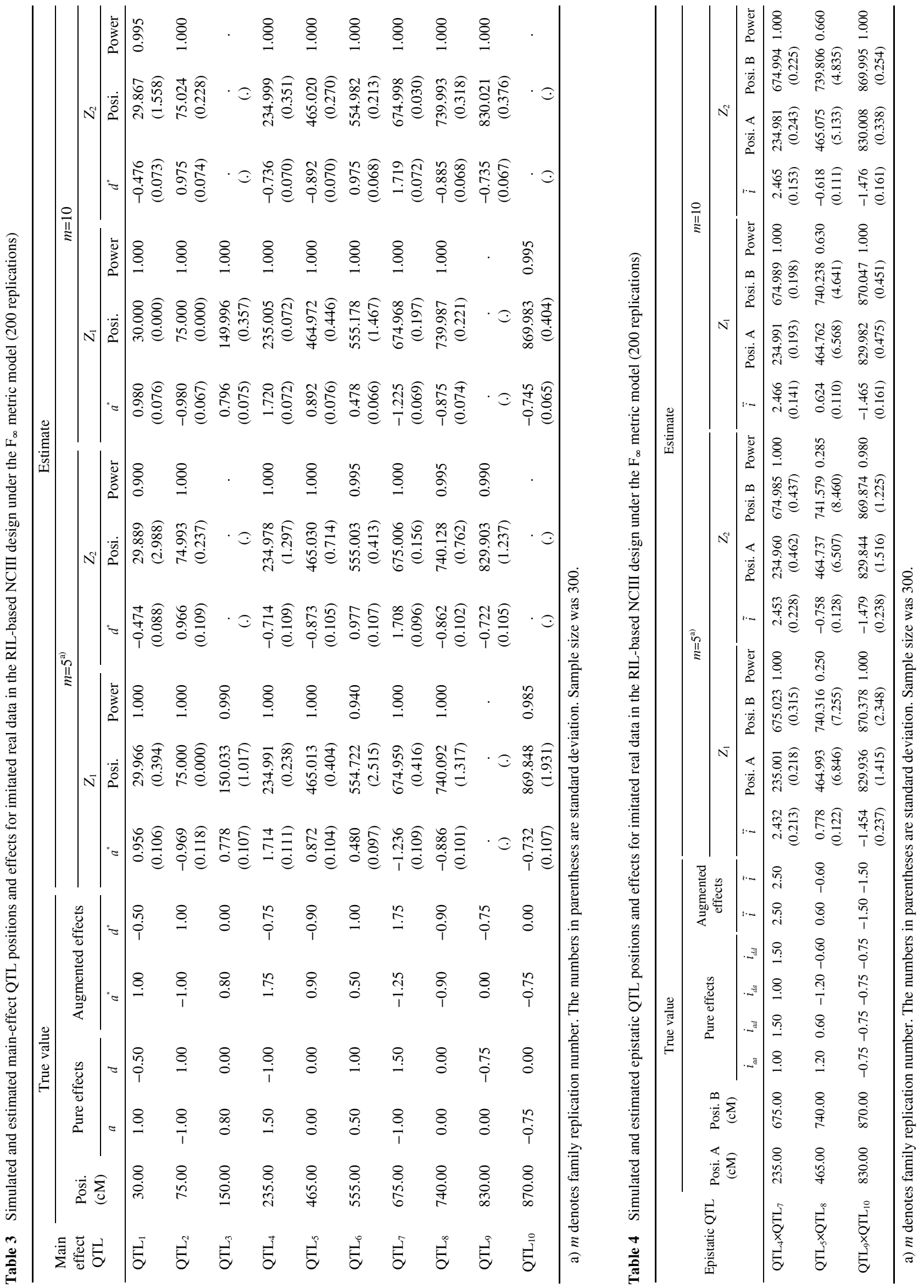

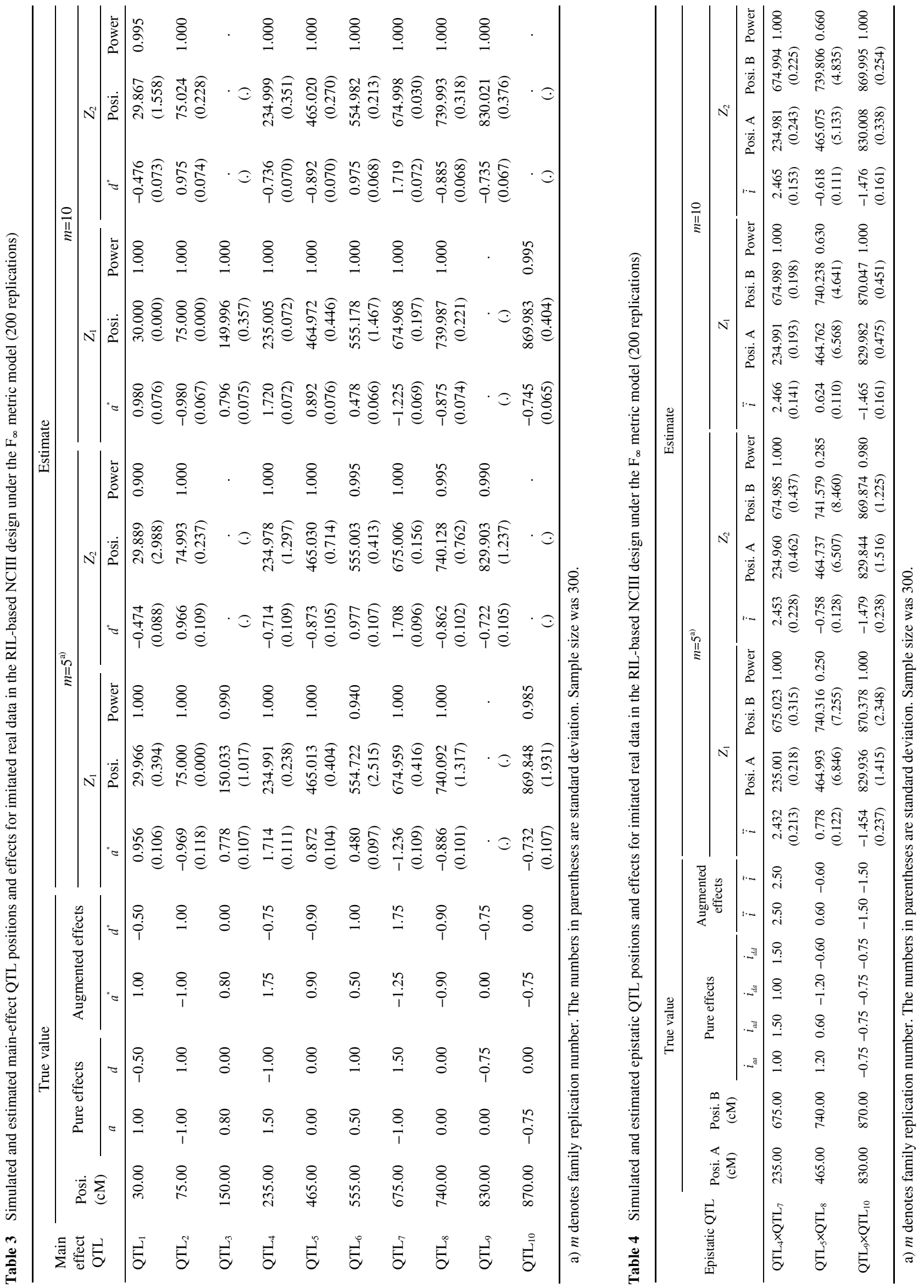

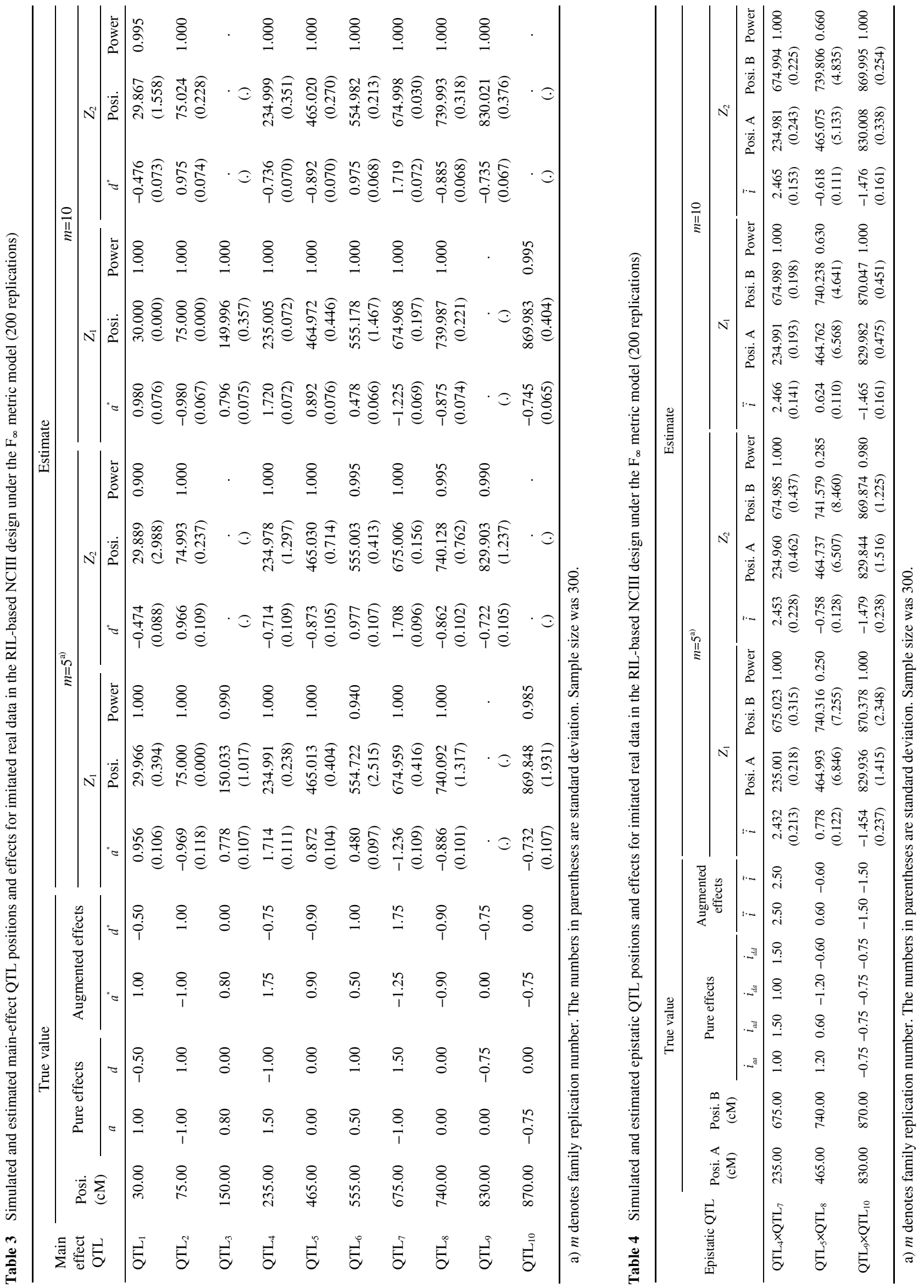

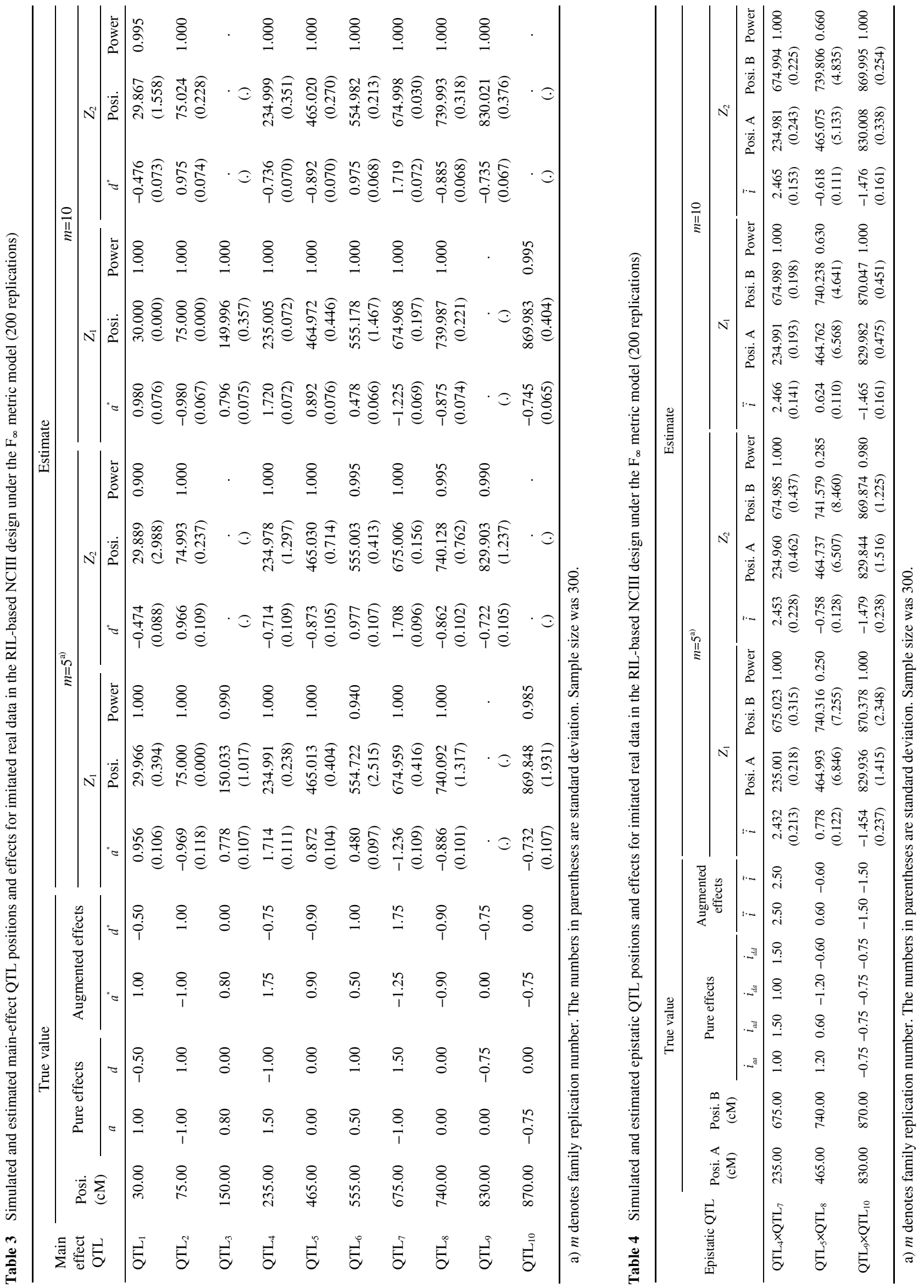

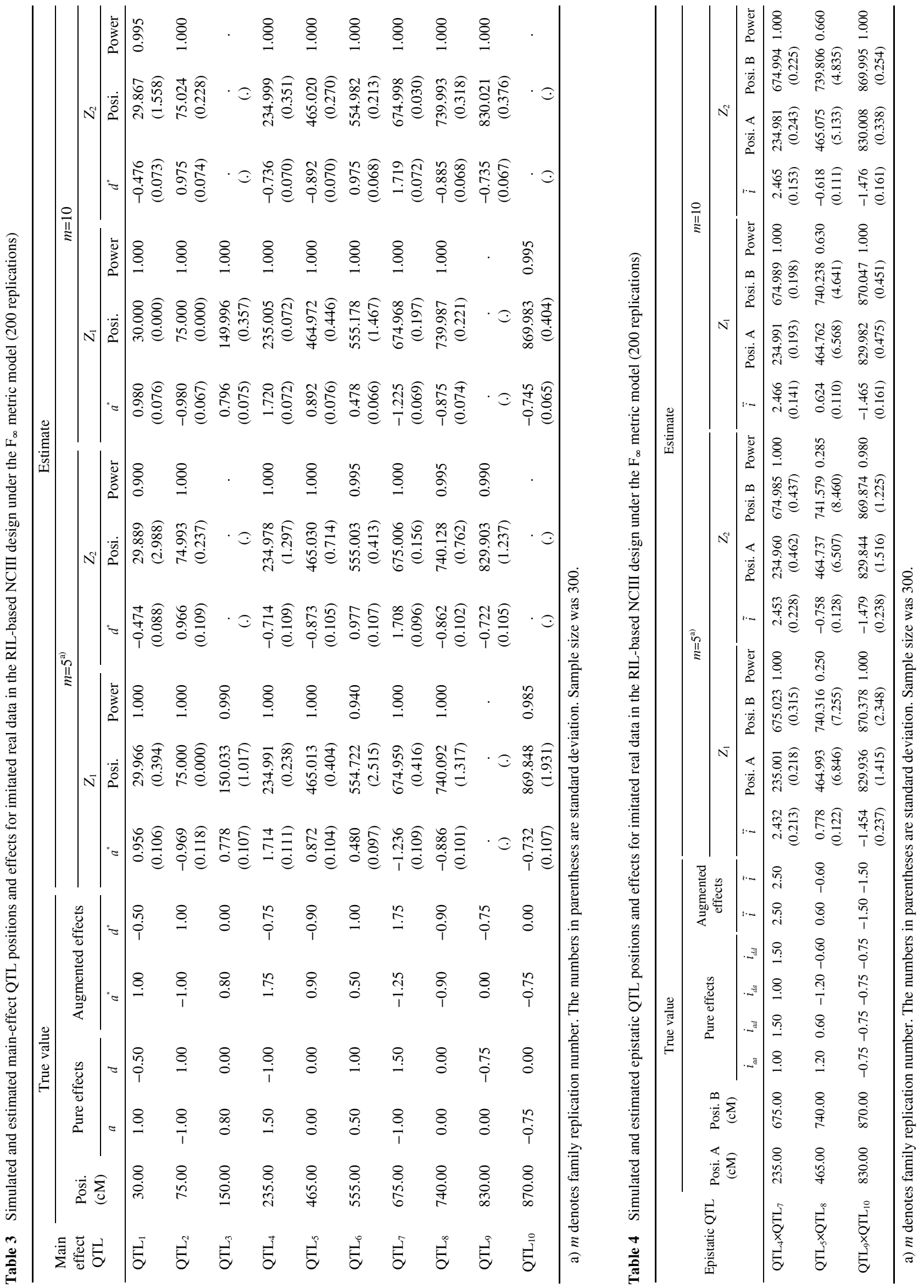




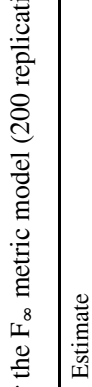

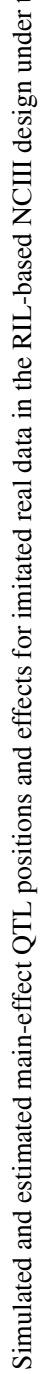

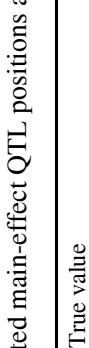

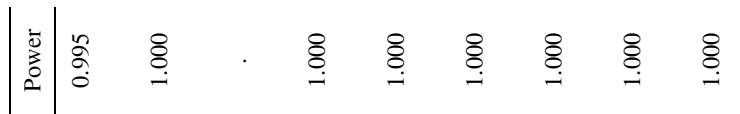

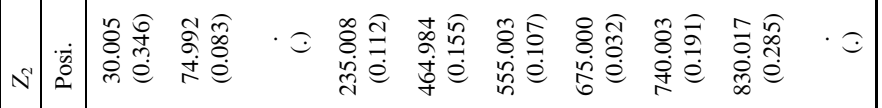

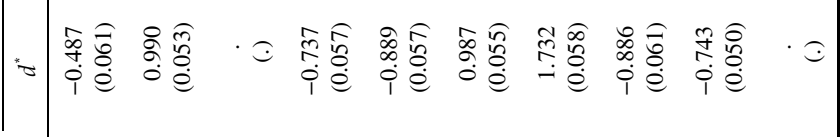

$\stackrel{ }{ }$

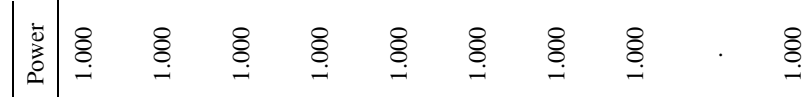

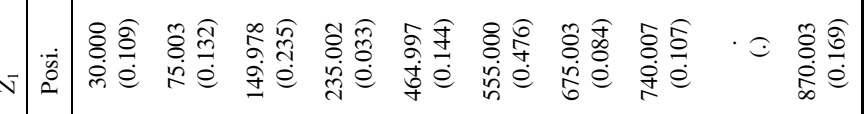

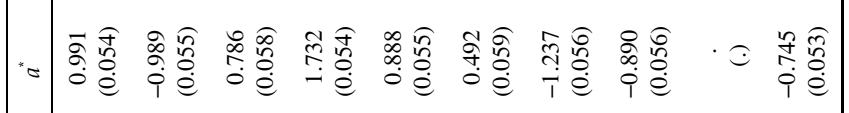

1.

言紊

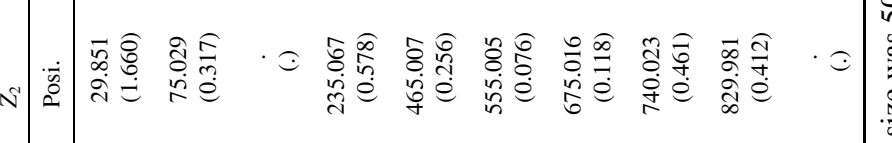

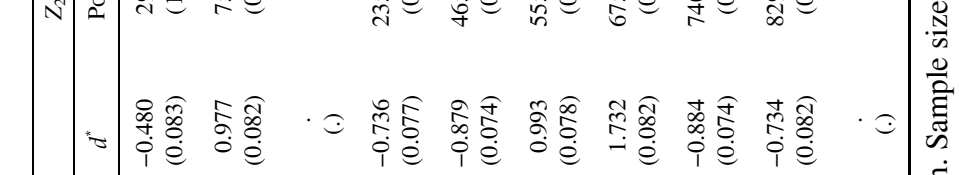

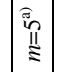

$\sqrt{\mathrm{g}}$

.

$\circ$

ळ.

\section{\&}

\section{1)}

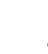

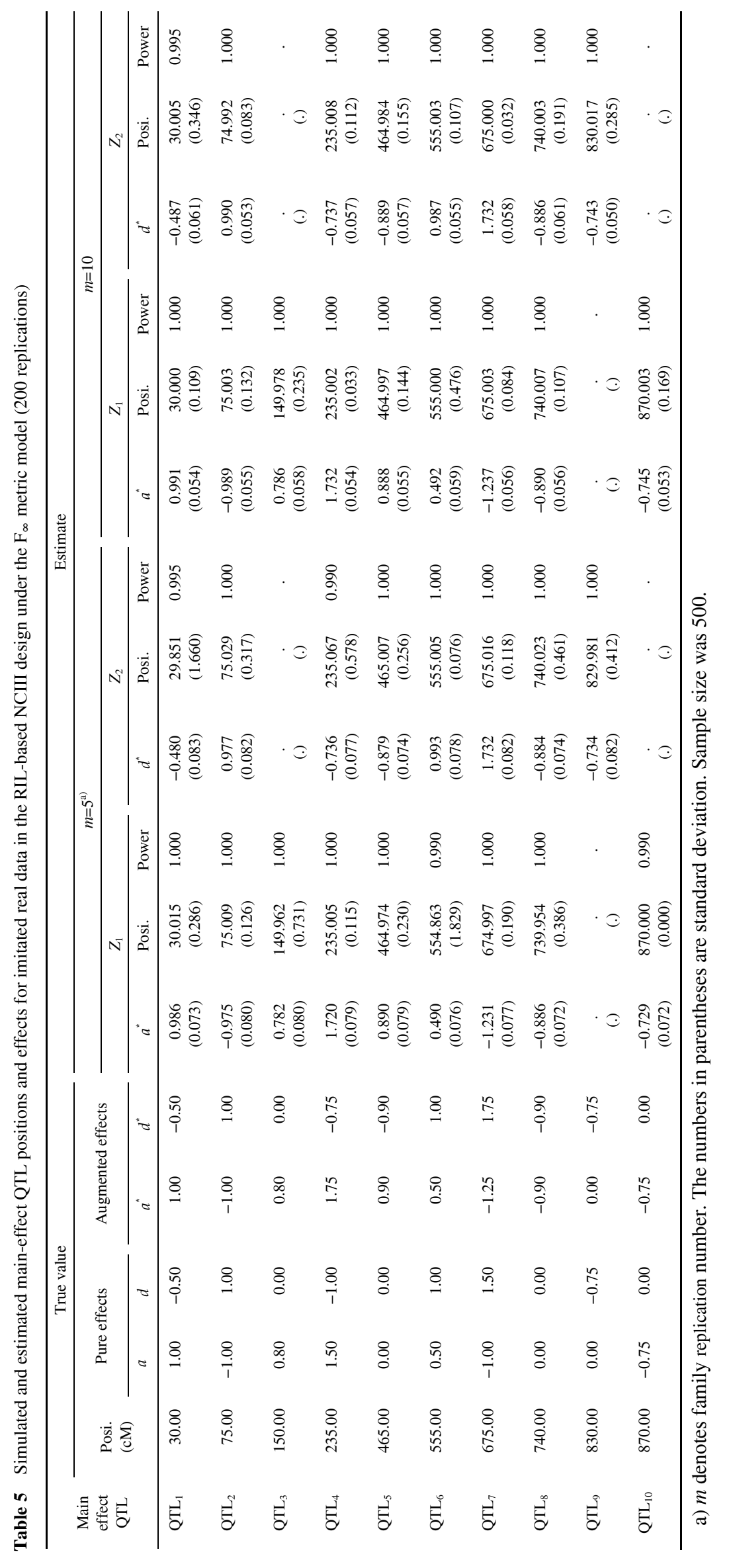

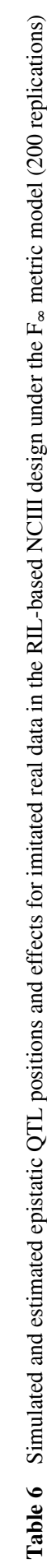


design. In the first step, with $Z_{1}$ and $Z_{2}$ the augmented main and epistatic effects were estimated, and with $Z_{3}$ three pure epistatic effects were obtained. In the second step, the three pure epistatic effects obtained in the first step were integrated with the augmented epistatic and main effects for the further estimation of all other pure effects.

Although the exploitation of heterosis in plant breeding is very successful, its genetic basis has been debated for more than 100 years and is still not completely resolved $[12,19,30]$. The genetic analysis of QTL can provide both molecular and practical understanding of this key phenomenon $[8,15,19,28]$. It should be noted that not all QTL detected in these studies contribute to trait heterosis $[14,25,43]$. In fact, two ways are tried to infer heterotic loci. The first one is based on the type of gene action. The additive QTL do not contribute to heterosis, while the non-additive QTL, including dominant, overdominant, and epistatic do contribute $[12,15,19,22,24,25,28]$. The second one is to use the measurements of heterosis as the data input to perform QTL analysis [13-15,24-26,30,43]. QTL effects for $Z_{1}$ and $Z_{2}$ in the RIL-based NCIII design provide estimates of the augmented effects $a^{*}$, which measures the net contribution of the QTL to the parental difference, and $d^{*}$, which measures the net contribution of the QTL to midparent heterosis [14]. Thus, QTL detected in the analysis of $Z_{2}$ data may be directly associated with trait heterosis, and the augmented dominance ratio $\left(\mathrm{ADR}=d^{*} /\left|a^{*}\right|\right)$ may measure the type of heterotic QTL.

This work was supported by the National Basic Research Program of China (2011CB109306), a Project Funded by the Priority Academic Program Development of Jiangsu Higher Education Institutions, the National Natural Science Foundation of China (30900842, 31000666) and the NAU Youth Sci-Tech Innovation Fund (KJ08001).

1 Shull G H. The composition of a field of maize. Am Breeders Assoc Rep, 1908, 4: 296-301

2 Goldman I L. From out of old fields comes all this new corn: An historical perspective on heterosis in plant improvement. In: Lamkey K, Staub J, eds. Concepts and Breeding of Heterosis in Crop Plants. Madison, W I: CSSA Special Publication, 1998. No. 25. 1-12

3 Filho J B M. Inbreeding depression and heterosis. In: Coors J G, Pandey S, eds. The Genetics and Exploitation of Heterosis in Crops. Madison, WI: ASA-CSSA-SSSA Societies, 1999. 69-80

4 Chen Z J. Molecular mechanisms of polyploidy and hybrid vigor. Trends Plant Sci, 2010, 15: 57-71

5 Darwin C R. The Effects of Cross- and Self-Fertilization in the Vegetable Kingdom. London: John Murray, 1876

6 Duvick D N. Biotechnology in the 1930s: The development of hybrid maize. Nat Rev Genet, 2001, 2: 69-74

7 Birchler J A, Yao H, Chudalayandi S, et al. Heterosis. Plant Cell, 2010, 22: 2105-2112

8 Lippman Z B, Zamir D. Heterosis: Revisiting the magic. Trends Genet, 2007, 23: 60-66

9 Comstock R E, Robinson H F. The components of genetic variance in populations of biparental progenies and their use in estimating the average degree of dominance. Biometrics, 1948, 4: 254-266

10 Comstock R E, Robinson H F. Estimation of average dominance of genes. In: Gowen J W, ed. Heterosis. Ames, IA: Iowa State College Press, 1952. 494-516

11 Cockerham C C, Zeng Z B. Design III with marker loci. Genetics,
1996, 143: 1437-1456

$12 \mathrm{Lu} \mathrm{H}$, Romero-Severson J, Bernardo R. Genetic basis of heterosis explored by simple sequence repeat markers in a random-mated maize population. Theor Appl Genet, 2003, 107: 494-502

13 Syed N H, Chen Z J. Molecular marker genotypes, heterozygosity and genetic interactions explain heterosis in Arabidopsis thaliana. Heredity, 2005, 94: 295-304

14 Melchinger A E, Utz H F, Piepho H P, et al. The role of epistasis in the manifestation of heterosis: A systems-oriented approach. Genetics, 2007, 177: 1815-1825

15 Li L, Lu K, Chen Z, et al. Dominance, overdominance and epistasis condition the heterosis in two heterotic rice hybrids. Genetics, 2008, 180: $1725-1742$

16 Garcia A A F, Wang S, Melchinger A E, et al. Quantitative trait loci mapping and the genetic basis of heterosis in maize and rice. Genetics, 2008, 180: 1707-1724

17 Schön C C, Dhillon B S, Utz H F, et al. High congruency of QTL positions for heterosis of grain yield in three crosses of maize. Theor Appl Genet, 2010, 120: 321-332

18 Kearsey M J, Pooni H S. The Genetical Analysis of Quantitative Traits. London: Chapman and Hall, 1996

19 Stuber C W, Lincoln S E, Wolff D W, et al. Identification of genetic factors contributing to heterosis in a hybrid from two elite maize inbred lines using molecular markers. Genetics, 1992, 132: 823-839

20 Tanksley S D, Medina-Filho H, Rick C M. Use of naturally-occurring enzyme variation to detect and map genes controlling quantitative traits in an interspecific backcross of tomato. Heredity, 1982, 49: 11-25

21 Lander E S, Botstein D. Mapping Mendelian factors underlying quantitative traits using RFLP linkage maps. Genetics, 1989, 121: 185-199

22 Xiao J, Li J, Yuan L, et al. Dominance is the major genetic basis of heterosis in rice as revealed by QTL analysis using molecular markers. Genetics, 1995, 140: 745-754

23 Wang D L, Zhu J, Li Z K, et al. Mapping QTLs with epistatic effects and QTLxenvironment interactions by mixed model approaches. Theor Appl Genet, 1999, 99: 1255-1264

24 Li Z K, Luo L J, Mei H W, et al. Overdominant epistatic loci are the primary genetic basis of inbreeding depression and heterosis in rice. I. Biomass and grain yield. Genetics, 2001, 158: 1737-1753

25 Luo L J, Li Z K, Mei H W, et al. Overdominant epistatic loci are the primary genetic basis of inbreeding depression and heterosis in rice. II. Grain yield components. Genetics, 2001, 158: 1755-1771

26 Mei H W, Li Z K, Shu Q Y, et al. Gene actions of QTLs affecting several agronomic traits resolved in a recombinant inbred rice population and two backcross populations. Theor Appl Genet, 2005, 110: 649-659

27 Zeng Z B. Precision mapping of quantitative trait loci. Genetics, 1994 136: $1457-1468$

28 LeDeaux J R, Graham G I, Stuber C W. Stability of QTLs involved in heterosis in maize when mapped under several stress conditions. Maydica, 2006, 51: 151-167

29 Frascaroli E, Canè M A, Pè M E, et al. QTL detection in maize testcross progenies as affected by related and unrelated testers. Theor Appl Genet, 2009, 118: 993-1004

30 Frascaroli $\mathrm{E}$, Canè $\mathrm{M}$ A, Landi $\mathrm{P}$, et al. Classical genetic and quantitative trait loci analyses of heterosis in a maize hybrid between two elite inbred lines. Genetics, 2007, 176: 625-644

31 Kao C H, Zeng Z B, Teasdale R. Multiple interval mapping for quantitative trait loci. Genetics, 1999, 152: 1203-1216

$32 \mathrm{Xu} \mathrm{S}$. An expectation-maximization algorithm for the Lasso estimation of quantitative trait locus effects. Heredity, 2010, 105: 483-494

33 Kao C H, Zeng Z B. Modeling epistasis of quantitative trait loci using Cockerham's model. Genetics, 2002, 160: 1243-1261

34 Yang R C. Epistasis of quantitative trait loci under different gene action models. Genetics, 2004, 167: 1493-1505

35 Zeng Z B, Wang T, Zou W. Modeling quantitative trait loci and interpretation of models. Genetics, 2005, 169: 1711-1725

36 Melchinger A E, Utz H F, Schön C C. Genetic expectations of quantitative trait loci main and interaction effects obtained with the triple testcross design and their relevance for the analysis of heterosis. Ge- 
netics, 2008, 178: 2265-2274

37 Wang H, Zhang Y M, Li X, et al. Bayesian shrinkage estimation of QTL parameters. Genetics, 2005, 170: 465-480

38 Zhang Y M, Xu S. A penalized maximum likelihood method for estimating epistatic effects of QTL. Heredity, 2005, 95: 96-104

39 Zhang J, Yue C, Zhang Y M. Bias correction for estimated QTL effects using the penalized maximum likelihood method. Heredity, 2012, 108: 396-402

$40 \mathrm{Xu} \mathrm{S}$. An empirical Bayes method for estimating epistatic effects of quantitative trait loci. Biometrics, 2007, 63: 513-521
41 He X H, Zhang Y M. A complete solution for dissecting pure main and epistatic effects of QTL in triple testcross design. PLoS ONE, 2011, 6: e24575

42 Li H H, Ribaut J M, Li Z L, et al. Inclusive composite interval mapping (ICIM) for digenic epistasis of quantitative traits in biparental populations. Theor Appl Genet, 2008, 116: 243-260

43 Hua J P, Xing Y Z, Wei W R, et al. Single-locus heterotic effects and dominance by dominance interactions can adequately explain the genetic basis of heterosis in an elite rice hybrid. Proc Natl Acad Sci USA, 2003, 100: 2574-2579

Open Access This article is distributed under the terms of the Creative Commons Attribution License which permits any use, distribution, and reproduction in any medium, provided the original author(s) and source are credited.

\section{Supporting Information}

Supplemental Information $\mathbf{A}$ The expected genetic values of $Z_{1 i}$ and $Z_{2 i}$ under the $\mathrm{F}_{\infty}$ and the $\mathrm{F}_{2}$ metric models in the RIL-based NCIII design.

Table S1 Genetic constitutions of $L_{1 i}$ and $L_{2 i}$ families derived from the RIL-based NCIII design

Table S2 Expected genetic values of $Z_{1 i}=\bar{L}_{1 i}+\bar{L}_{2 i}$ and $Z_{2 i}=\bar{L}_{1 i}-\bar{L}_{2 i}$ under the Fo metric model in the RIL-based NCIII design

Table S3 Expected genetic values of $Z_{1 i}=\bar{L}_{1 i}+\bar{L}_{2 i}$ and $Z_{2 i}=\bar{L}_{1 i}-\bar{L}_{2 i}$ under the $\mathrm{F}_{2}$ metric model in the RIL-based NCIII design

Supplemental Information B Statistical genetic models for mapping heterotic QTL in the RIL-based NCIII design under the $\mathrm{F}_{2}$ metric model.

Table S4 Heritability (\%) of each main and epistatic QTL for $L_{1}$ and $L_{2}$ populations in the simulated RIL-based NCIII design under both the $\mathrm{F}_{\infty}$ and $\mathrm{F}_{2}$ metric models

Table S5 Simulated and estimated main-effect QTL positions and effects for imitated real data in the RIL-based NCIII design under the $F_{2}$ metric model (200 replications)

Table S6 Simulated and estimated epistatic QTL positions and effects for imitated real data in the RIL-based NCIII design under the $F_{2}$ metric model (200 replications)

Table S7 Simulated and estimated main-effect QTL positions and effects for imitated real data in the RIL-based NCIII design under the $\mathrm{F}_{2}$ metric model (200 replications)

Table S8 Simulated and estimated epistatic QTL positions and effects for imitated real data in the RIL-based NCIII design under the $F_{2}$ metric model (200 replications)

Table S9 False positive rate $\left(\times 10^{-4}\right)$ for the analysis of $Z_{1}$ and $Z_{2}$ datasets in the simulated RIL-based NCIII design under both the $\mathrm{F}_{\infty}$ and $\mathrm{F}_{2}$ metric models

The supporting information is available online at csb.scichina.com and www.springerlink.com. The supporting materials are published as submitted, without typesetting or editing. The responsibility for scientific accuracy and content remains entirely with the authors. 